\title{
Single-nucleus sequencing reveals enriched expression of genetic risk factors sensitises Motor Neurons to degeneration in ALS
}

Francesco Limone ${ }^{1,2,3,10}$, Daniel Mordes ${ }^{1,2,4,10}$, Alexander Couto ${ }^{1}$, Olli Pietiläinen ${ }^{1,2,5}$, Brian J. Joseph ${ }^{1}$, Aaron Burberry $^{1,2,9}$, Sulagna Dia Ghosh ${ }^{1,2,6}$, Daniel Meyer ${ }^{2,6}$, Melissa Goldman ${ }^{2,6}$, Laura Bortolin ${ }^{2,6}$, Inma Cobos ${ }^{4}$, Martine Therrien $^{2,7}$, Beth Stevens ${ }^{2,7}$, Irena Kadiu ${ }^{8}$, Steven A. McCarroll ${ }^{2,6}$ \& Kevin Eggan ${ }^{1,2, *}$

${ }^{1}$ Department of Stem Cell and Regenerative Biology, Harvard University, Cambridge, MA, USA. ${ }^{2}$ Stanley Centre for Psychiatric Research, Broad Institute of MIT and Harvard, Cambridge, MA, USA. ${ }^{3}$ Hubrecht Institute for Developmental Biology and Stem Cell Research, Utrecht, The Netherlands. ${ }^{4}$ Department of Pathology, Massachusetts general Hospital, Boston, MA, USA. ${ }^{5}$ Neuroscience Center, Helsinki Institute of Life Science, University of Helsinki, Helsinki, Finland. ${ }^{6}$ Department of Genetics, Harvard Medical School, Boston MA, USA. 'Boston Children's Hospital, F.M. Kirby Neurobiology Center, Boston, MA, USA. ${ }^{7}$ Neuroscience Therapeutic Area, New Medicines, UCB Pharma, Braine-l'Alleud, Belgium. ${ }^{9}$ Department of Pathology, School of Medicine, Case Western Reserve University, Cleveland, OH, USA. ${ }^{10}$ These authors contributed equally: Francesco Limone, Daniel Mordes. *e-mail: eggan@mcb.harvard.edu

Amyotrophic Lateral Sclerosis (ALS) is a fatal neurodegenerative disorder characterised by a progressive loss of motor function. While it is known that the eponymous spinal sclerosis observed upon autopsy is the result of Cortico-Spinal Motor Neuron (CSMN) degeneration, it remains unclear why this neuronal subtype is selectively affected. To understand the unique molecular properties that sensitise deep-layer CSMNs to ALS, we performed RNA sequencing of 79,169 single nuclei from the frontal cortex of patients and controls. In unaffected individuals, we found that expression of ALS risk genes was most significantly enriched only in $\mathrm{THY} 1^{+}$presumptive CSMNs and not in other cortical cell types. In patients, these genetic risk factors, as well as additional genes involved in protein homeostasis and stress responses, were significantly induced in $\mathrm{THY} 1^{+} \mathrm{CSMNs}$ and a wider collection of deep layer neurons, but not in neurons with more superficial identities. Examination of oligodendroglial and microglial nuclei also revealed patient-specific gene expression changes We show microglial alterations can in part be explained by interactions with degenerating neurons. Overall, our findings suggest the selective vulnerability of CSMNs is due to a "first over the line" mechanism by which their intrinsic molecular properties sensitise them to genetic and mechanistic contributors to degeneration.

Amyotrophic Lateral Sclerosis (ALS) is characterised by the selective degeneration of both cortical-spinal and spinal motor neurons ${ }^{1}$. Although specific genetic causes of ALS have been identified, most cases are sporadic and have no family history of disease ${ }^{2,3}$. Bulk RNA-sequencing of post-mortem brain tissues has begun to identify gene expression alterations in both sporadic and familial forms of the disease ${ }^{4-7}$. One likely contributor of these alterations in gene expression is the aggregation and nuclear clearance of TAR DNA-binding protein-43 (TDP-43), which is found in the brain and spinal cord of over $95 \%$ of cases $^{8}$. However, the tissue level gene-expression analysis that has been reported to date has left uncertainty concerning the way in which distinct subtypes of neurons, including corticospinal motor neurons (CSMNs), are altered in the disease. Furthermore, it is increasingly understood that non-neuronal, glial cells are important modulators of neuronal degeneration, but it remains unclear how transcripts in these cell types are modulated in $\mathrm{ALS}^{9-12}$.

Methods to measure transcript abundance at a single-cell level have rapidly advanced and their application to nuclei from human post-mortem brain tissue has provided new insights into how individuals brain cell types are altered in Multiple Sclerosis (MS) ${ }^{13,14}$ and Alzheimer's disease (AD) $)^{15,16}$. Here, we report findings from RNA sequencing of single nuclei isolated from sporadic ALS and control pre-frontal cortex. Analyses of these data identify pathways altered by ALS in individual classes of cells and suggest a molecular explanation for the selective sensitivity of corticospinal motor neurons to degeneration.

\section{Profiling of ALS frontal cortex by single-nucleus RNA-sequencing}

To better understand factors that might contribute to the specific degeneration of classes of deep layer excitatory neurons, including CSMNs, we used single nucleus RNA sequencing to profile frontal cortex grey matter from 9 sporadic (sALS) patients and 8 age-matched controls with no known neurological disease using Drop-seq ${ }^{17}$. After screening for RNA quality, barcoded libraries from 119,510 individual nuclei, from 8 individuals were analysed ( $n=5$ sALS, $n=3$ Control) (Fig. 1a, Extended Data Table 1). Further quality control yielded 79,169 nuclear libraries (barcodes) with a mean 
of 1269 genes and 2026 unique molecular identifiers (UMIs) (Extended Data Fig. 1a-c). We used Seurat ${ }^{18}$, a single-cell analysis $\mathrm{R}$ package, to cluster and annotate nuclear libraries according to canonical markers of brain cell types: excitatory and inhibitory neurons, oligodendrocytes, oligodendrocyte progenitor cells (OPCs), microglia, astrocytes, and endothelial cells (Extended Data Figure 1d-f). The observed cell type distribution corresponded to previous studies ${ }^{19}$ and enabled robust categorization for downstream analysis. The cellular distribution was homogeneous between sexes and individuals, except for a modest decrease in the number of astrocytes in ALS samples (Extended Data Fig. 1g,h).

\section{Elevated expression of ALS-FTD risk genes in a specific class of CSMNs}

We first asked whether analysis of expression patterns of ALS genetic risk factors (Extended Data Fig. 2a) in our single nucleus dataset could provide insights into why certain cell types, including CSMNs, are more sensitive to degeneration. We began by computing a "module score" for the expression of this set of risk genes in the different cell types defined above. To this end, we generated a standardised z-score for the expression of each risk gene, summed it up as a total module score for the risk gene set and normalised this score with transcript abundance from a randomly selected, comparable set of genes ${ }^{20}$. Here, a positive score indicates higher expression of this risk gene set in a specific cell type compared to the average expression of the module across the collection of cell types in consideration. We also computed parallel module scores for gene lists compiled from latest GWAS for neurological disorders that also affect the cortex: $\mathrm{AD}^{21,22}$ and $\mathrm{MS}^{23}$ (Fig. 1a, Extended Data Table 2). Interestingly, we did not observe a clear enrichment for expression of ALS risk factors in any single broadly defined cell type (Fig. 1b). However, we did find enriched expression of AD and MS genetic risk factors in microglia in our dataset, as predicted by previous studies ${ }^{21-23}$ (Fig. 1c,d).

We next wondered whether combining the gene expression of all cortical excitatory neurons into a single profile might have prevented us from identifying enrichment of ALS risk gene expression in individual excitatory neuronal sub-types. TO identify these excitatory neuronal subtypes, we further examined 32,810 likely excitatory neuron nuclei by unbiased clustering and identified seven groups (Exc0-6) that expressed known markers of different cortical layers equally distributed in our patient/control cohort (Extended Data. Fig. 2b-e). Analysis of the ALS genetic risk factors in these cells showed a positive score in THY1-expressing neurons, subgroup Exc1 and no other excitatory sub-type (Normalised Enrichment Score=1.834) (Fig. 1e, Extended Data Fig. 2f). We observed no excitatory neuronal sub-type specific enrichment for $\mathrm{AD}$ and MS risk gene modules (Fig. 1f,g). THY1 is specifically enriched in human cortical layer $5^{13}$ and widely used as an expression marker for $\mathrm{CSMNs}^{13,24}$. Interestingly, neurons expressing upper layer marker CUX1 (Exc0) presented a lowerthan-expected expression of these genes (NES $=$-1.730) (Extended Data Fig. 2g). These findings were notable given the selective degeneration of CSMNs in ALS and findings from human samples ${ }^{25}$ and mouse models ${ }^{26}$ that suggest that superficial excitatory neuronal types have a lower propensity for pathologically accumulating TDP-43 relative to their deep layer counterparts.

\section{Distinct alterations in superficial and deep-layer neurons}

We next examined how the enriched expression of ALS-FTD genes relates to changes that occur in excitatory neurons in response to ALS. We conducted differential gene expression (DGE) analysis between neurons from patients and controls, across all excitatory neurons and within each excitatory subtype (Fig. 2a). To compare these signatures, we selected genes significantly upregulated in patients globally (DGEall) and within each subgroup (DGE0-6), calculated module-scores for each set and investigated whether certain neuronal subtypes might have similar responses to ALS (Extended Data Table 3). This analysis showed a correlation between scores in groups expressing markers of lower layers (Exc1,4,5,6) and the global transcriptomic changes identified in patients (Fig. 2b), suggesting that pathological changes in the lower cortical layers are driving the observed alterations. For instance, groups expressing deep-layer CSMNs markers (THY1-Exc1, FEZF2-Exc5) shared many upregulated genes with each other and with the more global excitatory signature. Strikingly, genes upregulated in upper layers of the cortex (CUX1-Exc0), a region relatively spared of TDP-43 pathology, largely lacked these similarities (Extended Data Fig. 3a). 
Subsequent Gene Ontology (GO) analysis showed that DEGs in CUX1-cells were associated with synaptic biology (Fig.2c). In contrast, DEGs identified in THY1-cells were connected to cellular stresses previously associated with $\operatorname{ALS}^{1,2}$ (Fig. 2d) and many were shared with transcriptional changes identified in patients' excitatory cells as a whole (Extended Data Figure 3b). Combining differentially expressed genes with protein-protein interaction data suggested coordinated alterations in the expression of genes that function in ribosomal, mitochondrial, protein folding, and protein degradation pathways including the proteasome and the lysosome (Fig. 2e, Extended Data Fig.3c-4). Interestingly, these pathways were specifically upregulated in neurons of deeper cortical layers rather than upper layer (Extended Data Fig. 3e).

We next asked if we could model aspects of these changes in vitro using neurons derived from human Pluripotent Stem Cells (hPSC) (Extended Data Fig. 5a). To recapitulate proteostatic stress we applied MG132, a proteasome inhibitor, to neurons ${ }^{27}$ which was sufficient to induce nuclear loss of TDP-43, early hallmark of ALS (Extended Data Fig. 5b,c). Subsequent RNA-sequencing of these neurons showed widespread transcriptomic changes after treatment, with many upregulated genes shared between stressed hPSC-neurons and neurons from sALS patients, especially proteasome subunits and heat-shock response-associated chaperonins (Extended Data Fig. 5d-f). GO analysis of 114 shared alterations confirmed the upregulation of proteasome processes and chaperone complexes and suggests a connection to neurodegeneration in ALS (Extended Data Fig. 5g). These findings show that proteasome inhibition can orchestrate alterations like those observed in deep layer neurons from ALS patients, underscoring those alterations in neuronal gene expression in ALS may in part be due to inhibition of proteostatic processes.

\section{Oligodendroglial respond to neuronal stress with a neuronally-engaged state}

CSMNs are long-projection neurons that reach into the spinal cord and are dependent on robust axonal integrity ${ }^{28}$, also changes in white matter and myelination have been associated with ALS patients ${ }^{11}$. We therefore analysed nuclei from cells involved in myelination. The 19,151 nuclei from oligodendroglia were clustered in five groups: one of OPCs - Oliglia3, and four of oligodendrocytes Oliglia0,1,2,4 (Fig. 3a-c, Extended Data Fig. 6a). We noted a significant depletion of ALS-nuclei in Oliglia0 whereas Oliglia1 and Oliglia4 were enriched in patients (Fig. 3d, Extended Data Fig. 6b-d). GO analysis for genes enriched in each group compared to others, revealed that Control-enriched Oliglia0 was characterised by terms connected to oligodendrocyte development and myelination and expressed higher levels of myelinating genes, e.g. CNP, OPALIN, MAG (Fig. 3e, Extended Data Fig. 7a,b). Conversely, ALS-enriched Oliglial show terms for neurite morphogenesis, synaptic organization and higher expression of postsynaptic genes $D L G 1, D L G 2$, GRID2 (Fig. 3f, Extended Data Fig. 7c,d).

Global differential gene expression analysis supports a shift from a myelinating to a neuronallyengaged state with upregulation of genes involved in synapse modulation and decrease of masterregulators of myelination, as confirmed by GO analysis (Fig. 3g-i, Extended Data Fig. 7f-i). Loss of myelination is exemplified by the expression of G-protein coupled receptors (GPRCs) that mark developmental milestones: GPR56, expressed in $\mathrm{OPCs}^{29}$, and GPR37, expressed in myelinating cells $\mathrm{s}^{30}$, were lowly expressed in ALS-enriched subgroups and globally downregulated (Extended Data Fig. 7e). Impaired myelination is consistent with previous studies identifying demyelination in sALS patients ${ }^{11}$.

To explore the relevance of these changes, we compared our study with published reports that identified shifts in oligodendrocytes ${ }^{14}$. We investigated the correlation of gene modules from Jäkel et al. ${ }^{14}$ in our study, revealing that Control-enriched Oliglia0 most closely resembled highly myelinating, $O P A L I N^{+}$cells from Jäkel (Extended Data Fig. 8a,b), while ALS-enriched Oliglia1 and Oliglia4 aligned to not-actively myelinating Jäkel1 (Extended Data Fig. 8c,d), with a high degree of shared genes (Extended Data Fig. 8e-h, Extended Data Table 4). The data so far shows how activation of stress pathways in deep layer neurons is accompanied by a shift in oligodendrocytes from active myelination to oligo-to-neuron contact. This shift, that in MS is associated with replacement of myelin at lesions, has an opposite response in ALS, where we observed a more "neuro-supportive" state (Fig. 3j).

Microglial activation is characterised by an ALS-specific endo-lysosomal response

Mouse models ${ }^{31}$, patient samples ${ }^{6}$ and ALS-related genes function in myeloid cells ${ }^{32-34}$ have demonstrated the importance of microglia as modifiers of disease, so we interrogated changes in this 
cell type. In the 1,452 nuclei we examined from microglia (Fig. 4a, Extended Data Fig. 9a), we identified 159 genes upregulated in patients and, remarkably, with many associated with endocytosis and exocytosis (e.g. TREM2, ASAH1, ATG7, SORL1, CD68). (Fig. 4b). Several of these genes were also associated with microglial activation (CTSD) and other neurodegenerative disorders (APOE) (Fig. $4 \mathrm{c}, \mathrm{d})$. Interestingly, several genes genetically associated with fALS were upregulated: OPTN, $S Q S T M 1 / p 62, G R N$ (Fig. 4e). GO analysis for upregulated genes indicated activation of endo-lysosomal pathways, secretion and immune cells degranulation which have been previously proposed to occur in myeloid cells in $\mathrm{ALS}^{33,34}$ (Fig. 4f,g). Further subclustering identified three groups: homeostatic Micro0, "Disease Associated Microglia"-like Micro1, and cycling Micro2 (Extended Data Fig. 9b-d). Notably, genes that characterised Micro1 were also upregulated in sALS (Extended Data Fig. 9e,f), with downregulation of homeostatic genes and upregulation of reactive pathways (Extended Data Fig. 9g-j).

To identify modulators of this signature, we used the Connectivity Map (CMap) pipeline ${ }^{35}$, which contains gene expression data of 9 human cell lines treated with thousands of perturbations and allows association between a given transcriptomic signature and a specific perturbation. This analysis revealed that genes dysregulated in sALS microglia positively correlated with regulators of cell cycle and senescence, KLF6 and CDKN1A/p21, suggesting an exhaustion of microglial proliferation might be occurring in ALS. On the other hand, we found a negative correlation with a type I-interferon-associated response (IFNB1), which is targeted in treatments for other neurological diseases to reduce inflammation ${ }^{35}$ (Extended Data Fig. 10a). Given the strong signature of homeostatic stress identified in deep layer neurons, we wondered whether changes seen in microglia might be caused by interactions with degenerating neurons. To test this idea, we separately differentiated microglia-like cells (iMGLs) ${ }^{36}$ and neurons (piNs) ${ }^{37}$ from hPSCs, triggered neuronal apoptosis and then introduced apoptotic neurons to iMGLs in culture (Extended Data Fig. 10b-c). Quantitative assessment of representative transcripts by RT-qPCR confirmed that apoptotic neurons lead to the significant upregulation of genes involved in the endo-lysosomal trafficking pathways identified in microglia from ALS patients (Extended Data Fig. 10d) suggesting that microglial changes are, at least in part, a response to degenerating neurons in sALS.

We next asked whether the microglial changes that we found were a general response to neuronal disease or restricted to ALS. By comparing our results with published snRNA-seq studies on human microglia in $\mathrm{AD}^{15}$ and $\mathrm{MS}^{38}$, we identified that dysregulation of lipid metabolism (APOE, APOCl, $S P P 1)$ was a common feature, and that many genes associated with DAMs were shared between ALS and MS (GPNMB, CTSD, CPM, LPL) and ALS and AD (e.g. TREM2) (Fig. 4h). Genes specifically upregulated in ALS were related to vesicle trafficking, myeloid cell degranulation and the lysosome (e.g., SQSTM1, GRN, ASAH1, LRRK2, LGALS3). This evidence suggests the induction of a shared reactive state of microglia in neurodegenerative diseases through the TREM2/APOE axis. Yet in ALS neuronal death more specifically activates changes in transcripts connected to dysfunctional endolysosomal pathways.

\section{Discussion}

A key question in the study of neurological disease is why certain neuronal types are more or less susceptible to degeneration in a particular condition. In this study, we identified the enrichment for expression of ALS risk genes in a class of CSMNs, which suggests clear mechanisms for their sensitivity to degeneration in $\mathrm{ALS}^{39}$. First, our findings suggest that the higher expression of these risk factors renders CSMNs potentially more sensitive to gain-of-function mutant variants in ALSassociated genes than other neuronal sub-types. Secondly, it implies that these neurons may have a constitutively heightened need for expression of certain risk factor genes, which may be burned by rare heterozygous loss of function mutations or altered in expression by regulatory variants. Strikingly, this enrichment was not recapitulated for risk factors connected to AD and MS in our CSMNs data, we instead replicated to be more enriched for expression in microglia.

Additionally, we identified a broadly shared transcriptomic signature of induction of homeostatic stress pathways in specific classes of deep layer excitatory neurons. These alterations in translation, proteostasis and mitochondrial function have previously been implicated in mouse models of ALS ${ }^{1,2}$. Our study indicates aligned changes occurring in deep-layer neuronal cell classes and highlights their cell-type specificity of these alterations. Importantly, we used human neuronal models to test whether a subset of these changes in gene expression were likely to be direct result of proteasome inhibition and found this to be the case. 
Emerging studies have shown that glial cells are important disease modulators in ALS. For instance, defects in oligodendrocyte maturation and myelination are present in SOD1-G93A mice and removing toxic SOD1 from this lineage improves surviva $1^{11}$. In our study, we demonstrated that changes in expression of transcripts involved in oligodendrocyte differentiation, myelination and synapse organization occur in ALS and may therefore contribute to neuronal degeneration or alternatively may be a coordinated response to the disease. Additionally, the gene expression changes in this lineage in ALS appear to be in polar opposition to those described in $\mathrm{MS}^{14}$. Moreover, we revealed perturbations in key myelin-regulators, such as $O P A L I N, C N P$, and $M A G$, across multiple oligodendrocyte clusters but in these cells only, as opposed to $\mathrm{AD}$ where myelination-related changes were present across multiple cell types ${ }^{15}$.

The role that synaptic apparatus and myelin assume in modulating neuronal excitability raises the question as to how regulation of synaptic signalling by oligodendrocytes might benefit neuronal survival. These changes are especially interesting if coupled with our finding concerning the upregulation of synaptic transcripts here identified in $\mathrm{CUX1}^{+}$upper layer excitatory neurons and the documented loss of postsynaptic density molecules in CSMNs in $\mathrm{ALS}^{40}$ and might relate to the changes in physiology observed in patients ${ }^{41}$. These observations suggest a response of the Cortico-Spinal motor circuit that attempts to compensate for the loss of neuronal inputs to the spinal cord and suggests that shifting oligodendroglial states may complement efforts aimed to alter excitatory inputs into CSMNs ${ }^{41}$.

Finally, we found distinct transcriptional perturbations in ALS-associated microglia, particularly in endo-lysosomal pathways. We and others have implicated ALS-associated gene C9orf72 in endosomal trafficking and secretion in myeloid cells ${ }^{33,34}$ and the upregulation of lysosomal constituents, e.g. CTSD, was identified in this study and confirmed by others in patients ${ }^{42}$. Coupled with the upregulation of fALS/FTD-associated genes SQSTM1/p62, OPTN, TREM2 and GRN, this suggests a mechanistic convergence on vesicle trafficking and pro-inflammatory pathways that may initiate and/or exacerbate the homeostatic-to-DAM transition in ALS. This observation underlines that the clear enrichment of ALS-related genes we identify in CSMNs might not be the only genetic driver of the disease and could be coupled with processes engaging disease related genes in different cells, i.e. microglia. We also delineated changes in senescence and interferon-responsive genes, as confirmed by others in C9orf72-ALS ${ }^{43}$. Overall, differentially expressed transcripts in microglia had partial overlap with those in microglia surrounding amyloid plaques in $\mathrm{AD}^{15,16}$ and microglia associated with demyelinating lesions in $\mathrm{MS}^{38}$, suggesting that partially shared but not altogether identical pathways are engaged in these neurodegenerative diseases, which clearly warrants further study.

In summary, we show that CSMNs harbour significantly higher expression of a collection of genetic risk factors for ALS/FTD that are also expressed in other deep-layer neuronal cell types but are depleted in their expression in excitatory neurons with more superficial identities. We hypothesise that this intrinsically higher expression of disease-associated genes in putative CSMNs might be at the bottom of a "first over the line" mechanism leading to initial degeneration of this cell-type, followed by other "less-vulnerable" deep-layer neurons. Overall, our data suggests that these alterations in CSMNs and other deep layer cortical neurons may trigger a cascade of responses: superficial neurons upregulate synaptic genes potentially to supplement for lost inputs to the cord; oligodendroglia shift from a myelinating to a neuronally-engaged state; microglia activate a pro-inflammatory state in response to neuronal degeneration. Future investigations should consider how the individual alterations to distinct cell-types are ordered in disease processes and now that they are further elaborated, their relative importance in disease progression.

1 Taylor, J. P., Brown, R. H., Jr. \& Cleveland, D. W. Decoding ALS: from genes to mechanism. Nature 539, 197-206, doi:10.1038/nature20413 (2016).

2 Brown, R. H. \& Al-Chalabi, A. Amyotrophic Lateral Sclerosis. N Engl J Med 377, 162-172, doi:10.1056/NEJMra1603471 (2017).

3 Wainger, B. J. \& Lagier-Tourenne, C. Taking on the Elephant in the Tissue Culture Room: iPSC Modeling for Sporadic ALS. Cell Stem Cell 23, 466-467, doi:10.1016/j.stem.2018.09.015 (2018).

4 Prudencio, M. et al. Distinct brain transcriptome profiles in C9orf72-associated and sporadic ALS. Nat Neurosci 18, 1175-1182, doi:10.1038/nn.4065 (2015).

$5 \quad$ Mordes, D. A. et al. Dipeptide repeat proteins activate a heat shock response found in C9ORF72ALS/FTLD patients. Acta Neuropathol Commun 6, 55, doi:10.1186/s40478-018-0555-8 (2018). 
6 D'Erchia, A. M. et al. Massive transcriptome sequencing of human spinal cord tissues provides new insights into motor neuron degeneration in ALS. Sci Rep 7, 10046, doi:10.1038/s41598-017-10488-7 (2017).

7 Tam, O. H. et al. Postmortem Cortex Samples Identify Distinct Molecular Subtypes of ALS: Retrotransposon Activation, Oxidative Stress, and Activated Glia. Cell Rep 29, 1164-1177 e1165, doi:10.1016/j.celrep.2019.09.066 (2019).

8 Neumann, M. et al. Ubiquitinated TDP-43 in frontotemporal lobar degeneration and amyotrophic lateral sclerosis. Science 314, 130-133, doi:10.1126/science.1134108 (2006).

9 Suzuki, N. et al. The mouse C9ORF72 ortholog is enriched in neurons known to degenerate in ALS and FTD. Nat Neurosci 16, 1725-1727, doi:10.1038/nn.3566 (2013).

10 Ransohoff, R. M. How neuroinflammation contributes to neurodegeneration. Science 353, 777-783, doi:10.1126/science.aag2590 (2016).

11 Kang, S. H. et al. Degeneration and impaired regeneration of gray matter oligodendrocytes in amyotrophic lateral sclerosis. Nat Neurosci 16, 571-579, doi:10.1038/nn.3357 (2013).

12 Boillee, S. et al. Onset and progression in inherited ALS determined by motor neurons and microglia. Science 312, 1389-1392, doi:10.1126/science.1123511 (2006).

13 Schirmer, L. et al. Neuronal vulnerability and multilineage diversity in multiple sclerosis. Nature 573, 75-82, doi:10.1038/s41586-019-1404-z (2019).

14 Jakel, S. et al. Altered human oligodendrocyte heterogeneity in multiple sclerosis. Nature 566, 543-547, doi:10.1038/s41586-019-0903-2 (2019).

15 Mathys, H. et al. Single-cell transcriptomic analysis of Alzheimer's disease. Nature 570, 332-337, doi:10.1038/s41586-019-1195-2 (2019).

16 Zhou, Y. et al. Human and mouse single-nucleus transcriptomics reveal TREM2-dependent and TREM2independent cellular responses in Alzheimer's disease. Nat Med 26, 131-142, doi:10.1038/s41591-0190695-9 (2020).

17 Macosko, E. Z. et al. Highly Parallel Genome-wide Expression Profiling of Individual Cells Using Nanoliter Droplets. Cell 161, 1202-1214, doi:10.1016/j.cell.2015.05.002 (2015).

18 Stuart, T. et al. Comprehensive Integration of Single-Cell Data. Cell 177, 1888-1902 e1821, doi:10.1016/j.cell.2019.05.031 (2019).

19 Lake, B. B. et al. Integrative single-cell analysis of transcriptional and epigenetic states in the human adult brain. Nat Biotechnol 36, 70-80, doi:10.1038/nbt.4038 (2018).

20 Tirosh, I. et al. Dissecting the multicellular ecosystem of metastatic melanoma by single-cell RNA-seq. Science 352, 189-196, doi:10.1126/science.aad0501 (2016).

21 Kunkle, B. W. et al. Genetic meta-analysis of diagnosed Alzheimer's disease identifies new risk loci and implicates Abeta, tau, immunity and lipid processing. Nat Genet 51, 414-430, doi:10.1038/s41588-0190358-2 (2019).

22 Jansen, I. E. et al. Genome-wide meta-analysis identifies new loci and functional pathways influencing Alzheimer's disease risk. Nat Genet 51, 404-413, doi:10.1038/s41588-018-0311-9 (2019).

23 International Multiple Sclerosis Genetics, C. Multiple sclerosis genomic map implicates peripheral immune cells and microglia in susceptibility. Science 365, doi:10.1126/science.aav7188 (2019).

24 Ozdinler, P. H. et al. Corticospinal motor neurons and related subcerebral projection neurons undergo early and specific neurodegeneration in hSOD1G(9)(3)A transgenic ALS mice. J Neurosci 31, 41664177, doi:10.1523/JNEUROSCI.4184-10.2011 (2011).

25 Nana, A. L. et al. Neurons selectively targeted in frontotemporal dementia reveal early stage TDP-43 pathobiology. Acta Neuropathol 137, 27-46, doi:10.1007/s00401-018-1942-8 (2019).

26 Porta, S. et al. Patient-derived frontotemporal lobar degeneration brain extracts induce formation and spreading of TDP-43 pathology in vivo. Nat Commun 9, 4220, doi:10.1038/s41467-018-06548-9 (2018).

27 Klim, J. R. et al. ALS-implicated protein TDP-43 sustains levels of STMN2, a mediator of motor neuron growth and repair. Nat Neurosci 22, 167-179, doi:10.1038/s41593-018-0300-4 (2019).

28 Tomassy, G. S. et al. Distinct profiles of myelin distribution along single axons of pyramidal neurons in the neocortex. Science 344, 319-324, doi:10.1126/science.1249766 (2014).

29 Giera, S. et al. The adhesion G protein-coupled receptor GPR56 is a cell-autonomous regulator of oligodendrocyte development. Nat Commun 6, 6121, doi:10.1038/ncomms7121 (2015).

30 Yang, H. J., Vainshtein, A., Maik-Rachline, G. \& Peles, E. G protein-coupled receptor 37 is a negative regulator of oligodendrocyte differentiation and myelination. Nat Commun 7, 10884, doi:10.1038/ncomms10884 (2016).

31 Keren-Shaul, H. et al. A Unique Microglia Type Associated with Restricting Development of Alzheimer's Disease. Cell 169, 1276-1290 e1217, doi:10.1016/j.cell.2017.05.018 (2017).

32 de Boer, A. S. et al. Genetic validation of a therapeutic target in a mouse model of ALS. Sci Transl Med 6, 248ra104, doi:10.1126/scitranslmed.3009351 (2014). 
Zhang, Y. et al. The C9orf72-interacting protein Smcr8 is a negative regulator of autoimmunity and lysosomal exocytosis. Genes Dev 32, 929-943, doi:10.1101/gad.313932.118 (2018).

34 Burberry, A. et al. C9orf72 suppresses systemic and neural inflammation induced by gut bacteria. Nature 582, 89-94, doi:10.1038/s41586-020-2288-7 (2020).

35 Subramanian, A. et al. A Next Generation Connectivity Map: L1000 Platform and the First 1,000,000 Profiles. Cell 171, 1437-1452 e1417, doi:10.1016/j.cell.2017.10.049 (2017).

36 Abud, E. M. et al. iPSC-Derived Human Microglia-like Cells to Study Neurological Diseases. Neuron 94, 278-293 e279, doi:10.1016/j.neuron.2017.03.042 (2017).

37 Nehme, R. et al. Combining NGN2 Programming with Developmental Patterning Generates Human Excitatory Neurons with NMDAR-Mediated Synaptic Transmission. Cell Rep 23, 2509-2523, doi:10.1016/j.celrep.2018.04.066 (2018).

38 Masuda, T. et al. Spatial and temporal heterogeneity of mouse and human microglia at single-cell resolution. Nature 566, 388-392, doi:10.1038/s41586-019-0924-x (2019).

39 Santillo, A. F. \& Englund, E. Greater loss of von Economo neurons than loss of layer II and III neurons in behavioral variant frontotemporal dementia. Am J Neurodegener Dis 3, 64-71 (2014).

40 Genc, B. et al. Apical dendrite degeneration, a novel cellular pathology for Betz cells in ALS. Sci Rep 7, 41765, doi:10.1038/srep41765 (2017).

41 Wainger, B. J. et al. Effect of Ezogabine on Cortical and Spinal Motor Neuron Excitability in Amyotrophic Lateral Sclerosis: A Randomized Clinical Trial. JAMA Neurol 78, 186-196, doi:10.1001/jamaneurol.2020.4300 (2021).

42 O'Rourke, J. G. et al. C9orf72 is required for proper macrophage and microglial function in mice. Science 351, 1324-1329, doi:10.1126/science.aaf1064 (2016).

43 McCauley, M. E. et al. C9orf72 in myeloid cells suppresses STING-induced inflammation. Nature 585, 96-101, doi:10.1038/s41586-020-2625-x (2020).

\section{Methods}

Human donor tissue. Post-mortem human cortical samples from ALS patients and age-matched controls were obtained at Massachusetts General Hospital using a Partners IRB approved protocol and stored at $-80^{\circ} \mathrm{C}$.

Isolation of nuclei. RNA quality of brain samples was assessed by running bulk nuclear RNA on an Agilent TapeStation for RIN scores. Extraction of nuclei from frozen samples was performed as previously described ${ }^{44}$. Briefly, tissue was dissected and minced with a razor blade on ice and then placed in $4 \mathrm{ml}$ ice-cold extraction buffer (Wash buffer (82 mM Na2SO4, $30 \mathrm{mM} \mathrm{K2SO4,} 5 \mathrm{mM} \mathrm{MgCl}$, $10 \mathrm{mM}$ glucose, and $10 \mathrm{mM}$ HEPES, pH adjusted to 7.4 with $\mathrm{NaOH}$ ) containing 1\% Triton X-100 and 5\% Kollidon VA64). Tissue was homogenized with repeated pipetting, followed by passing the homogenized suspension twice through a $261 / 2$ gauge needle on a 3 $\mathrm{ml}$ syringe (pre-chilled), once through a $20 \mu \mathrm{m}$ mesh filter, and once through a $5 \mu \mathrm{m}$ filter using vacuum. The nuclei were then diluted in $50 \mathrm{ml}$ ice-cold wash buffer, split across four $50 \mathrm{ml}$ tubes, and centrifuged at 500xg for 10 minutes at $4^{\circ} \mathrm{C}$. The supernatant was discarded, the nuclei pellet was resuspended in $1 \mathrm{ml}$ cold wash buffer.

10X loading and library preparation. Nuclei were DAPI-stained with Hoechst, loaded onto a hemacytometer, and counted using brightfield and fluorescence microscopy. The solution was diluted to $\sim 176$ nuclei/ul before proceeding with Drop-seq as described in ref. $15^{17}$. cDNA amplification was performed using around 6000 beads per reaction with 16 PCR cycles. The integrity of both the cDNA and fragmented libraries were assessed for quality control on the Agilent Bioanalyzer as in ef $^{45}$. Libraries were sequenced on a Nova-seq $\mathrm{S} 2$, with a $60 \mathrm{bp}$ genomic read. Reads were aligned to the human genome assembly (hg19). Digital Gene Expression files were generated with the Zamboni Drop-seq analysis pipeline, designed by the McCarroll group ${ }^{44}$.

Filtering of expression matrices and clustering of single nuclei. A single matrix for all samples was built by filtering any barcode with less than 400 genes and resulting in a matrix of 27,600 genes across 119,510 barcodes. This combined UMI matrix was used for downstream analysis using Seurat (v3.0.2) ${ }^{18}$. A Seurat object was created from this matrix by setting up a first filter of min.cells $=20$ per genes. After that, barcodes were further filtered by number of genes detected nFeature_RNA $>600$ and nFeature_RNA $<6000$. Distribution of genes and UMIs were used as parameters for filtering barcodes. The matrix was then processed via the Seurat pipeline: log-normalized by a factor of 10,000, followed by regressing out UMI counts (nCount_RNA), scaled for gene expression.

After quality filtering, 79,830 barcoes and 27,600 genes were used to compute SNN graphs and $t$-SNE projections using the first 10 statistically significant Principal Components. SNN-graphed $t$-SNE projection was used to determine minimum number of clusters obtain at resolution $=0.2$ (FindClusters). Broad cellular identities were assigned to groups on the basis of differentially expressed genes as calculated by Wilcoxon rank sum test in FindAllMarkers(min.pct $=0.25$, logfc.threshold $=0.25$ ). One subcluster with specifically high ratio of UMIs/genes 
was filtered out resulting in 79,169 barcodes grouped in 7 major cell types of the CNS: excitatory neurons, oligodendrocytes, inhibitory neurons, astrocytes, endothelial cells, microglia, oligodendrocyte progenitor cells (OPCs). Markers for specific cell types were identified in previously published human scRNAseq studies ${ }^{19}$.

Analysis of cellular subtypes were conducted by subsetting each group. Isolated barcodes were re-normalised and scaled and relevant PCs were used for re-clustering as a separate analysis. This newly scaled matrix was used for Differential Gene Expression analysis with parameters FindAllMarkers(min.pct $=0.10, \operatorname{logfc}$.threshold $=0.25$ ) and subclustering for identification of subgroups. Gene scores for different cellular subclusters were computed in each re-normalised, re-scaled sub-matrix using the AddModule function in Seurat v3.0.2.

Gene Ontology, Interactome and Gene Set Enrichment Analyses. For GO terms analysis, we selected statistically significant up-regulated or down-regulated genes identified in each subcluster as described before (adj p-values $<0.05, \mathrm{LFC}=2$ ). These lists were fed in the gProfiler pipeline ${ }^{46}$ with settings: use only annotated genes, g:SCS threshold of 0.05 , GO cellular components and GO biological processes $\left(26^{\text {th }}\right.$ of May $2020-9^{\text {th }}$ of December 2020), only statistically significant pathways are highlighted. For oligodendrocytes cells (Extended Data Fig.8) statistically significant up-regulated genes identified in each subcluster as described before (adj pvalues $<0.05, \mathrm{LFC}=2$ ) were used for synaptic specific Gene Ontology analysis using SynGO ${ }^{47}\left(12^{\text {th }}\right.$ of June 2020$)$. Interactome map was built using $\mathrm{STRING}^{48}$ protein-protein interaction networks, all statistically significant upregulated genes were used, 810 were identified as interacting partners using "experiments" as interaction sources and a high confidence threshold (0.700), only interacting partners are shown in Extended Data Figure 6. Gene Set Enrichment Analysis was performed using GSEA software designed by UC San Diego and the Broad Institute $(\mathrm{v} 4.0 .3)^{49}$. Briefly, gene expression matrices were generated in which for each subcluster each individual was a metacell, lists for disease-associated risk genes were compiled using available datasets (PubMed - ALSFTD - Supplementary Table 2) or recently published GWAS for $\mathrm{AD}^{21,22}$ and $\mathrm{MS}^{23}$.

Generation of Microglia-like Cells. Microglial-like cells were differentiated as described in Abud et al. ${ }^{36}$. Briefly, hPSCs were cultured in E8 medium (Stemcell technologies) on Matrigel (Corning), dissociated with Accutase (Stemcell technologies), centrifuged at 300xg for 5 minutes, resuspended in E8 medium with $10 \mu \mathrm{M}$ Y27632 ROCK Inhibitor, $2 \mathrm{M}$ cells are transferred to a low-attachment T25 flask in $4 \mathrm{ml}$ of medium and left in suspension for 24 hours. The first 10 days of differentiation are carried out in iHPC medium: IMDM (50\%, Stemcell technologies), F12 (50\%, Stemcell technologies), ITSG-X 2\% v/v (ThermoFisher), L-ascorbic acid 2Phosphate (64 ug/ml, Sigma), monothioglycerol (400 mM, Sigma), PVA (10 mg/ml; Sigma), Glutamax (1X, Stemcell technologies), chemically-defined lipid concentrate (1X, Stemcell technologies), non-essential amino acids (NEAA, Stemcell technologies). After 24h (day0), cells are collected and differentiation is started in iHPC medium supplemented with FGF2 (Peprotech, $50 \mathrm{ng} / \mathrm{ml}$ ), BMP4 (Peprotech, $50 \mathrm{ng} / \mathrm{ml}$ ), Activin-A (Peprotech, $12.5 \mathrm{ng} / \mathrm{ml}), \mathrm{Y}-27632$ ROCK Inhibitor $(1 \mu \mathrm{M})$ and $\mathrm{LiCl}(2 \mathrm{mM})$ and transferred in hypoxic incubator $\left(20 \% \mathrm{O}_{2}\right.$, $5 \% \mathrm{CO}_{2}, 37^{\circ} \mathrm{C}$ ). On day 2 , medium is changed to iHPC medium plus FGF2 (Peprotech, $50 \mathrm{ng} / \mathrm{ml}$ ) and VEGF (Peprotech, $50 \mathrm{ng} / \mathrm{ml}$ ) and returned to hypoxic conditions. On day4, cells are resuspended in iHPC medium supplemented with FGF2 (Peprotech, $50 \mathrm{ng} / \mathrm{ml}$ ), VEGF (Peprotech, $50 \mathrm{ng} / \mathrm{ml}$ ), TPO (Peprotech, $50 \mathrm{ng} / \mathrm{ml}$ ), SCF (Peprotech, $10 \mathrm{ng} / \mathrm{ml}$ ), IL-6 (Peprotech, $50 \mathrm{ng} / \mathrm{ml}$ ), and IL-3 (Peprotech, $10 \mathrm{ng} / \mathrm{ml}$ ) and placed into a normoxic incubator $\left(20 \% \mathrm{O}_{2}, 5 \% \mathrm{CO}_{2}, 37^{\circ} \mathrm{C}\right)$. Expansion of haematopoietic progenitors is continued by supplementing the flasks with $1 \mathrm{ml}$ of iHPC medium with small molecules every two days. On day10, cells are collected and filtered through a $40 \mu \mathrm{m}$ filter. The single cell suspension is counted and plated at 500,00 cells/well of a 6 well plate coated with Matrigel (Corning) in Microglia differentiation medium: DMEM/F12 (Stemcell technologies), ITS-G 2\%v/v (Thermo Fisher Scientific), B27 (2\%v/v, Stemcell technologies), N2 (0.5\%v/v, Stemcell technologies), monothioglycerol (200 mM, Sigma), Glutamax (1X, Stemcell technologies), NEAA (1X, Stemcell technologies), supplemented with M-CSF (25 ng/ml, Peprotech), IL-34 (100 ng/ml, Peprotech), and TGFb-1 (50 ng/ml, Peprotech). Induced Microglia-like cells (iMGLs) are kept in this medium for 20 days with change three times a week. On day 30, cells are collected and plated on poly-D-lysine/laminin coated dishes in Microglia differentiation medium supplemented with CD200 (100 ng/ml, Novoprotein) and CX3CL1 (100 ng/ml, PeproTech), M-CSF (25 ng/ml, PeproTech), IL-34 (100 ng/ml, PeproTech), and TGFb-1 (50 ng/ml, PeproTech) until day 40.

Feeding of apoptotic neurons to Microglia-like Cells. For feeding assays, neurons were generated from human iPSCs using an NGN2 overexpression system as described previously ${ }^{37}$. Day30 hiPSC-neurons "piNs" were treated with $2 \mu \mathrm{M} \mathrm{H}_{2} \mathrm{O}_{2}$ for 24 hours to induce apoptosis. Apoptotic neurons were gently collected from the plate and the medium containing the apoptotic bodies was transferred into wells containing day40 iMGLs. After 24 hours, iMGLs subjected to apoptotic neurons and controls were collected for RNA extraction.

RNA extraction and RT-qPCR analysis. RNA was extracted with the miRNeasy Mini Kit (Qiagen, 217004). cDNA was produced with iScript kit (BioRad) using $50 \mathrm{ng}$ of RNA. RT-qPCR reactions were performed in 
triplicates using $20 \mathrm{ng}$ of cDNA with SYBR Green (BioRad) and were run on a CFX96 Touch ${ }^{\mathrm{TM}}$ PCR Machine

Generation of hiPSC-derived neurons for bulk RNA sequencing. Human embryonic stem cells were cultured in mTESR (Stemcell technologies) on matrigel (Corning). Neurons were generated from HuES-3-Hb9:GFP based on the motor neuron differentiation protocol previously described ${ }^{27}$. Upon completion of the differentiation protocol, cells were sorted via flow-cytometry based on GFP signal intensity to yield GFP-positive neurons that were plated on PDL/laminin-coated plates (Sigma, Life technologies). Neurons were maintained in Neurobasal medium (Life Technologies) supplemented with N2 (Stemcell technologies), B27 (Life technologies), glutamax (Life technologies), non-essential amino acids (Life technologies), and neurotrophic factors (BDNF, GDNF, CNTF), and were grown for 28 days before the application of the proteasome inhibitors MG132 for 24 hrs. RNA was extracted using RNeasy Plus kit (Qiagen), libraries were prepared using the Illumina TruSeq RNA kit v2 according to the manufacturer's directions, and sequenced at the Broad Institute core with samples randomly assigned between two flow chambers. The total population RNA-seq FASTQ data was aligned against ENSEMBL human reference genome (build GRCh37/hg19) using STAR (v.2.4.0). Cufflinks (v.2.2.1) was used to derive normalized gene expression in fragments per kilo base per million (FPKM). The read counts were obtained from the aligned BAM-files in R using Rsubread. Differential gene expression was analyzed from the read counts in DESeq2 using a Wald's test for the treatment dosage and controlling for the sequencing flow cell.

Western blot analysis. For WB analyses, cells were lysed in RIPA buffer with protease inhibitors (Roche). After protein quantification by BCA assay (ThermoFisher), ten micrograms of proteins were preheated in Laemmli's buffer (BioRad), loaded in 4-20\% mini-PROTEAN ${ }^{\circledR}$ TGX ${ }^{\mathrm{TM}}$ precast protein gels (BioRad) and gels were transferred to a PDVF membrane. Membranes were blocked in Odyssey Blocking Buffer (Li-Cor) and incubated overnight at $4{ }^{\circ} \mathrm{C}$ with primary antibodies. After washing with TBS-T, membranes were incubated with IRDye ${ }^{\circledR}$ secondary antibodies (Li-Cor) for one hour and imaged with Odyssey® CLx imaging system (Li-Cor). List of primary antibodies can be found in Appendix.

Proteasome activity assay. Neurons were sorted in 96-wells plates and, after two weeks of maturation, treated for 24 hours. Cells were washed with 1xPBS, exposed to ProteasomeGlo® (Promega, G8660) and incubated for 30 minutes at RT. Fluorescence was measured using a Cytation ${ }^{\mathrm{TM}} 3$ reader (BioTek).

44 Krienen, F. M. et al. Innovations present in the primate interneuron repertoire. Nature 586, 262-269, doi:10.1038/s41586-020-2781-z (2020).

45 Saunders, A. et al. Molecular Diversity and Specializations among the Cells of the Adult Mouse Brain. Cell 174, 1015-1030 e1016, doi:10.1016/j.cell.2018.07.028 (2018).

46 Raudvere, U. et al. g:Profiler: a web server for functional enrichment analysis and conversions of gene lists (2019 update). Nucleic Acids Res 47, W191-W198, doi:10.1093/nar/gkz369 (2019).

47 Koopmans, F. et al. SynGO: An Evidence-Based, Expert-Curated Knowledge Base for the Synapse. Neuron 103, 217-234 e214, doi:10.1016/j.neuron.2019.05.002 (2019).

48 Szklarczyk, D. et al. STRING v11: protein-protein association networks with increased coverage, supporting functional discovery in genome-wide experimental datasets. Nucleic Acids Res 47, D607D613, doi:10.1093/nar/gky1131 (2019).

49 Subramanian, A. et al. Gene set enrichment analysis: a knowledge-based approach for interpreting genome-wide expression profiles. Proc Natl Acad Sci $U$ S A 102, 15545-15550, doi:10.1073/pnas.0506580102 (2005).

Acknowledgements. We thank the study participants and staff at Massachusetts Alzheimer's Disease Research Center (NIA P50 AG005134). We thank UCB Pharma for partially funding these studies. We would also like to thank Paul Tesar and his group for invaluable discussions on oligodendroglial biology.

Author contributions. This study was designed by F.L., D.M., S. M., K.E. and directed and coordinated by K.E. and S.M. F.L. performed bioinformatics analysis with the help of S.D.G., D.M. and D.M. under the supervision of K.E., B.S. and I.K. D.M. and I.C. supported obtaining post-mortem samples and carried out nuclei isolation and RNA-sequencing with M.G. and L.B. M.T., O.P., A.B., A.C. and B.J.J. performed bioinformatics analyses of the bulk RNA-sequencing from cells and helped with protein and RNA validation with cellular models. K.E. acquired primary funding.

Conflicting interests. IK is an employee at UCB Pharma and holds stock options.

Additional information. 


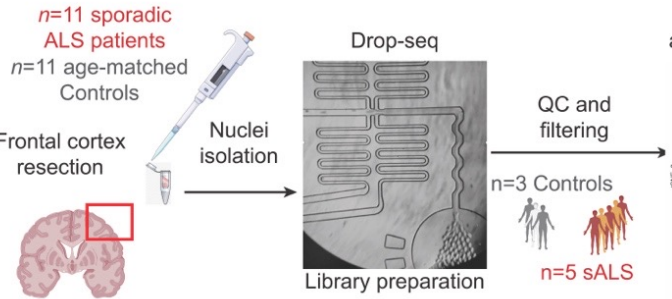

Broad cell-type annotation and gene expression analysis
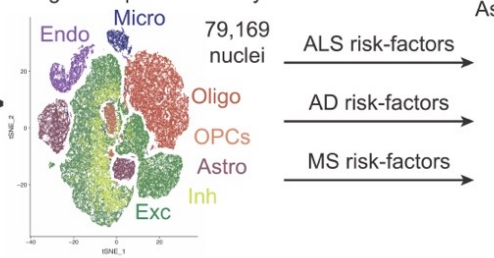

Assign expression z-score for each list of genetic risk factors per cell in SALS and Control

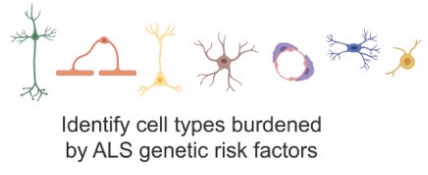

b ALS-FTD risk factors expression score in the cortex

C $\quad A D$ risk factors expression score in the cortex

d MS risk factors expression score in the cortex
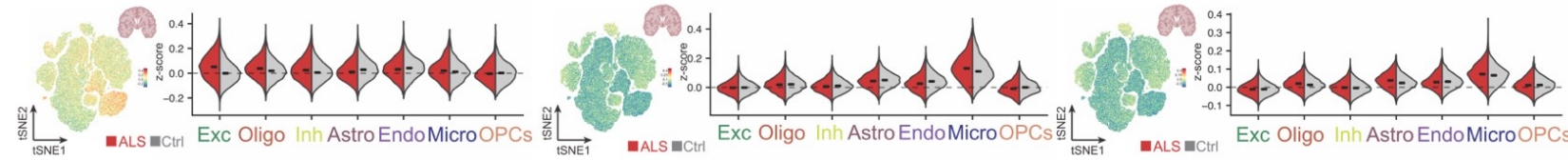

e

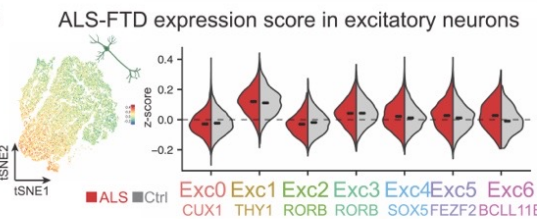

f

$A D$ expression score in excitatory neurons

g

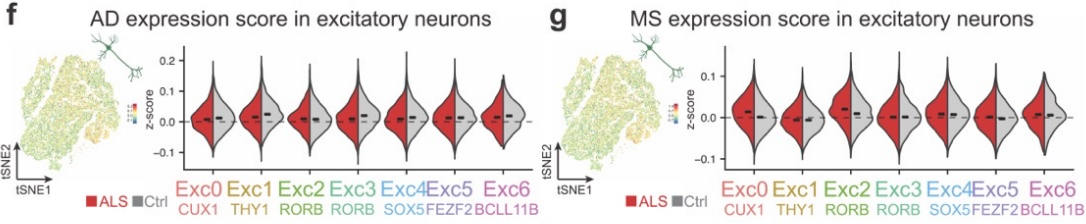

Fig. 1 Cellular susceptibility to ALS-FTD in the human cortex. a. Schematic diagram of workflow for isolation of nuclei from cortices of ALS patients and age-matched controls followed by single-cell RNA sequencing and assessment of expression of gene modules associated to neurodegenerative diseases. b-c. Violin plots and $t$-SNE projection for z-scores for expression of genes associated with the ALS-FTD (b), AD (c) and MS (d) in the different cell types identified in the cortex (bars denote median for each side of the violin plot). e-g. Violin plots and $t$-SNE projection for z-scores for expression of genes associated with the ALS-FTD (e), AD (f) and MS (g) in the different subtypes of excitatory neurons (bars denote median for each side of the violin plot). 


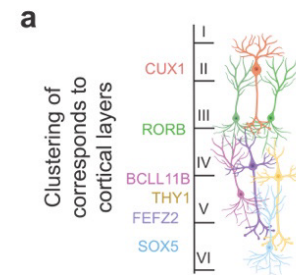

b

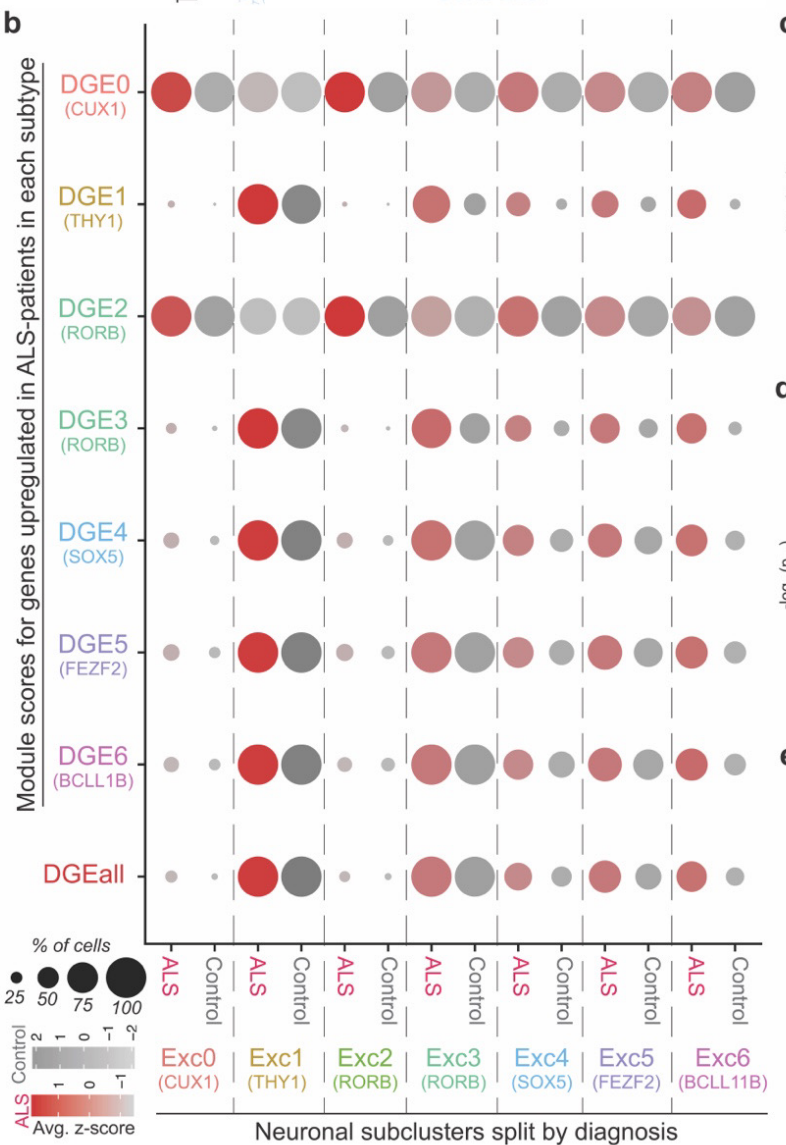

Upper layers

CUX1-Exc0

RORB-Exc2 Lower layers

RORB-Exc3 THY1-Exc1

FEFZ2-Exc5

BCLL11B-Exc5

SOX5-Exc4

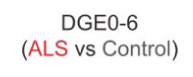

Differential Gene

Expression analysis within specific layers
wion

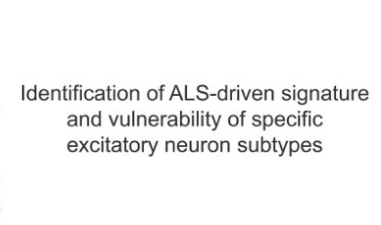

DGEall

(ALS vs Control)

Differential Gene Expression analysis
of all Exc neurons

${ }_{16}$ GO terms for genes upregulated in ALS CUX2 excitatory neurons (DGEO)

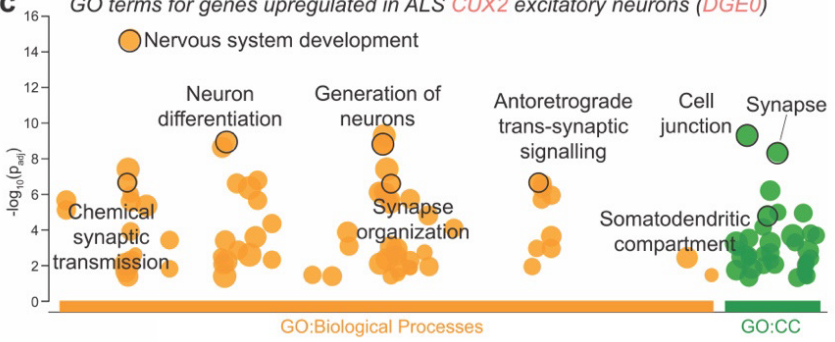

d GO terms for genes upregulated in ALS THY1 excitatory neurons (DGE1)

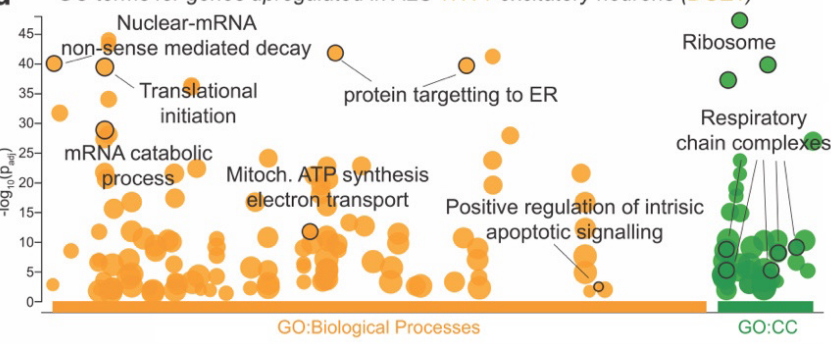

e Nuclear-mRNA nonsense-mediated decay GO::0000184 GO::0000184
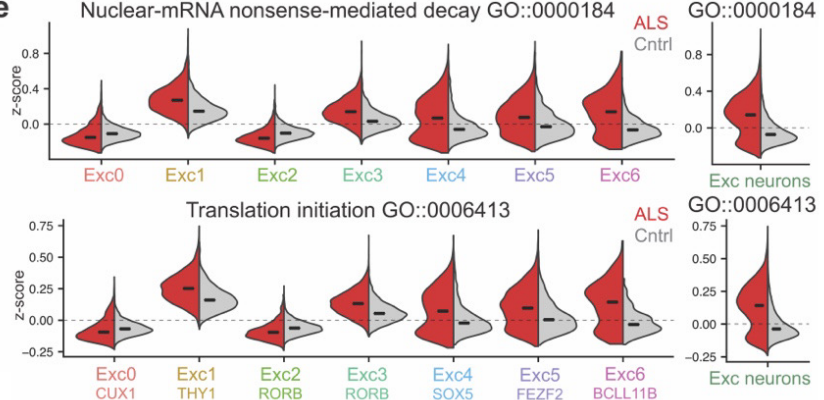

Fig. 2 Excitatory neurons from ALS cortex present increased expression of stress-related pathways. c. Schematic of Differential Gene Expression Analysis strategy b. Dotplot representing the scores for DEGenes upregulated in each subgroup of excitatory neurons (DGE0-1) and globally upregulated in all excitatory cells (DGEall). c. Gene Ontology analysis of terms for genes upregulated in CUX2-Exc0 group (DGE0), highlighted terms involved in synaptic biology $(\mathrm{CC}=$ Cellular Components $)$. d. Gene Ontology analysis for genes upregulated in THY1-Exc1 group (DGE1), highlighted terms are stress pathways involved in ALS pathology (CC=Cellular Components). e. Violin plots representing z-score for selected, statistically significant GO terms from analysis shown in $\mathrm{f}$, in each subgroup (left) and at the global level (right). 

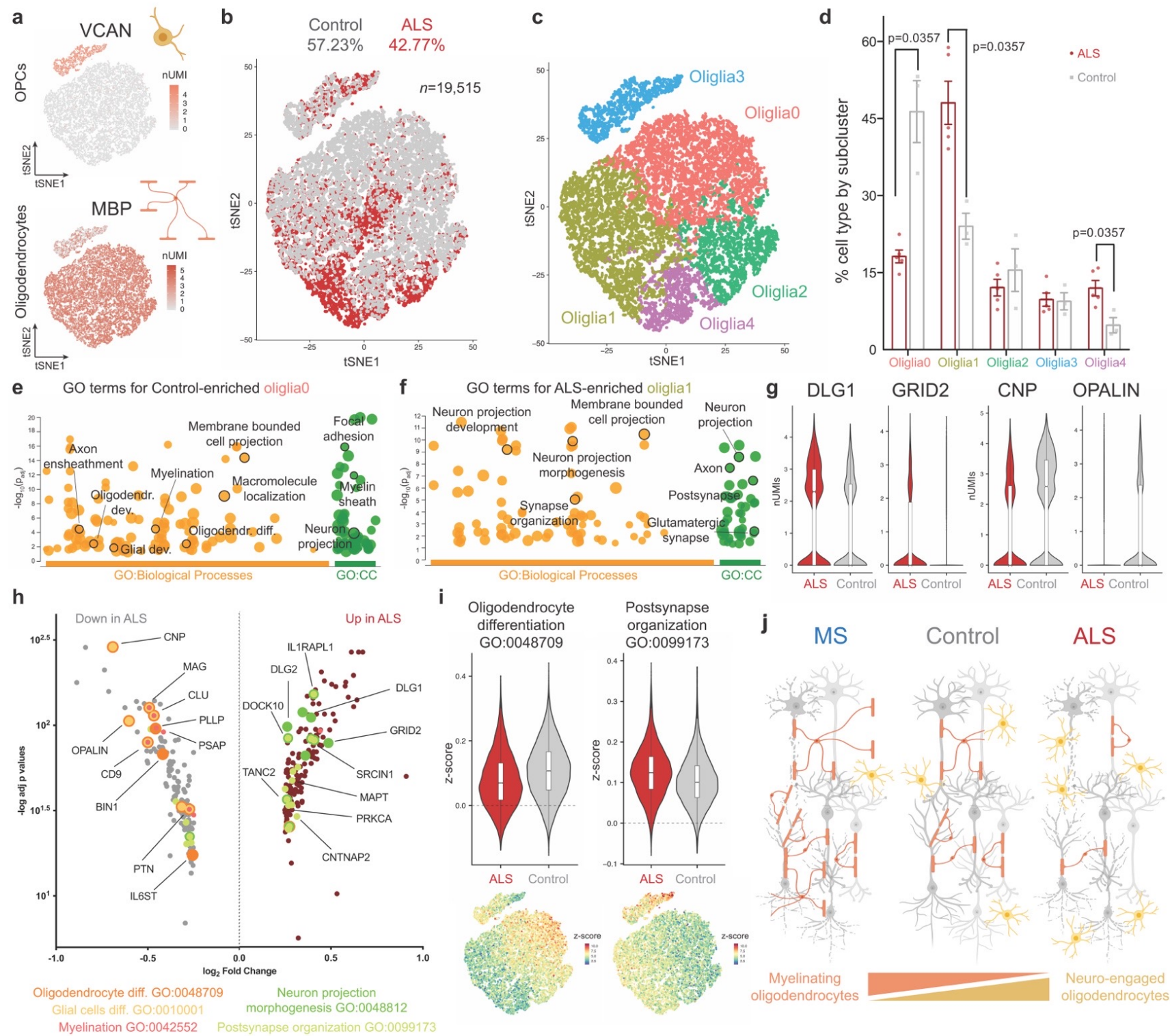

ALS

Fig. 3 In ALS, oligodendroglial cells decrease their myelinating machinery in favour of a neuro-supportive state. a. $t$-SNE projection of markers of OPCs and oligodendrocytes. b. $t$-SNE projection of oligodendroglial cluster (ALS $n=8,372$ nuclei, Control $n=11,168$ nuclei). c. $t$-SNE projection of subclusters identified within oligodendroglia. d. Distribution of subclusters by diagnosis. e. Gene Ontology analysis for genes characteristic of Control-enriched oliglia0, highlighted terms involved in myelination ( $\mathrm{CC}=$ Cellular Components). f. Gene Ontology analysis for genes characteristic of ALS-enriched oliglia1, highlighted terms involved in neuro-supportive functions $(\mathrm{CC}=\mathrm{Cellular}$ Components). g. Violin plots of representative genes for neuro-supportive functions (left) and myelination (right). $\mathbf{h}$. Volcano plot of differentially expressed genes in oligodendroglia. Highlighted genes identified in GO terms related to myelination (orange) and neuro-supportive functions (green). i. Violin plots representing z-score for selected GO terms and related $t$-SNE projection. j. Diagram illustrates proposed shift of oligodendrocytes states. 

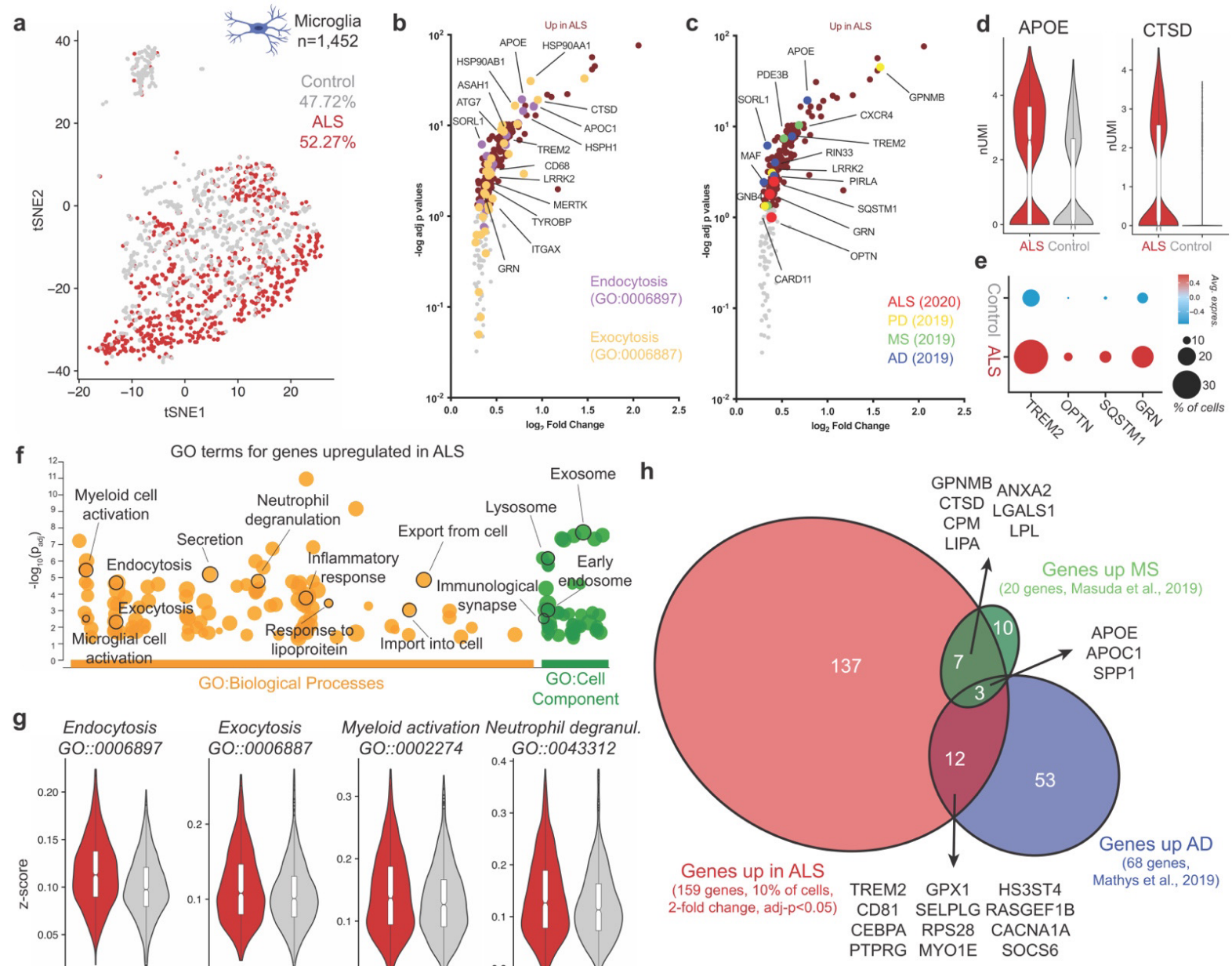

g Endocytosis
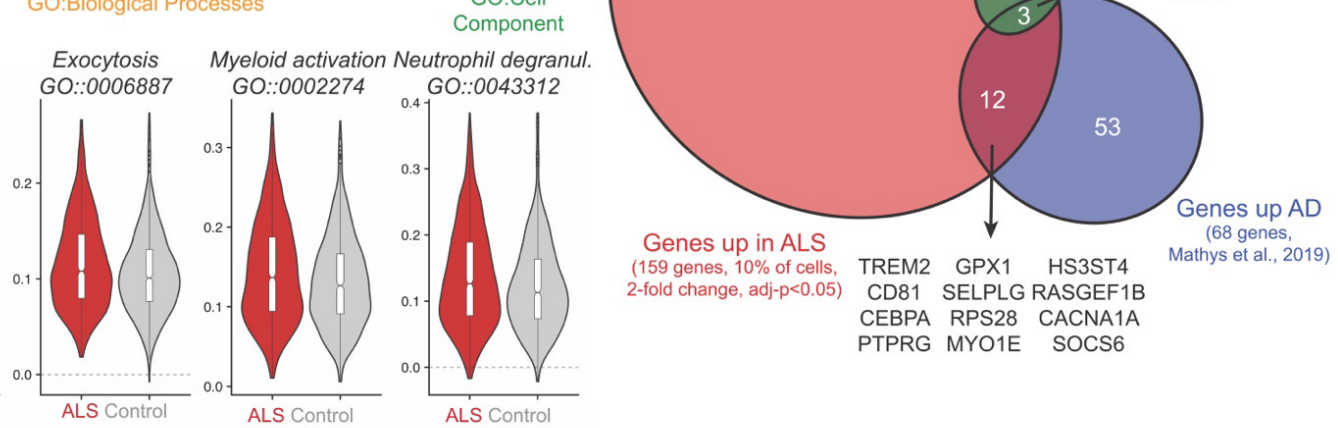

Fig. 4 Disease-Associated Microglia signature in ALS. a. $t$-SNE projection of microglia (ALS $n=759$ nuclei, Control $n=693$ nuclei). b,c. Volcano plot of genes upregulated in microglia from ALS. Genes identified in Gene Ontology terms for endocytosis and exocytosis highlighted in $\mathbf{b}$, genes associated to neurodegenerative diseases highlighted in c (ALS, PD - Parkinson's disease, MS, AD). d. Violin plots of representative genes upregulated in ALS patients associated with reactive microglia (geometric boxplots represent median and interquantile ranges). e. Dotplot representing expression of genes associated with ALS-FTD pathogenesis upregulated in microglia from patients. f. Gene Ontology analysis for genes upregulated in ALS microglia, highlighted terms involved in myeloid cells biology and/or pathogenesis of ALS. g. Violin plots representing z-score for selected, statistically significant GO terms from $4 \mathrm{f}$. h. Comparison of genes upregulated in microglia from ALS patients with genes upregulated in microglia in other neurodegenerative diseases. 

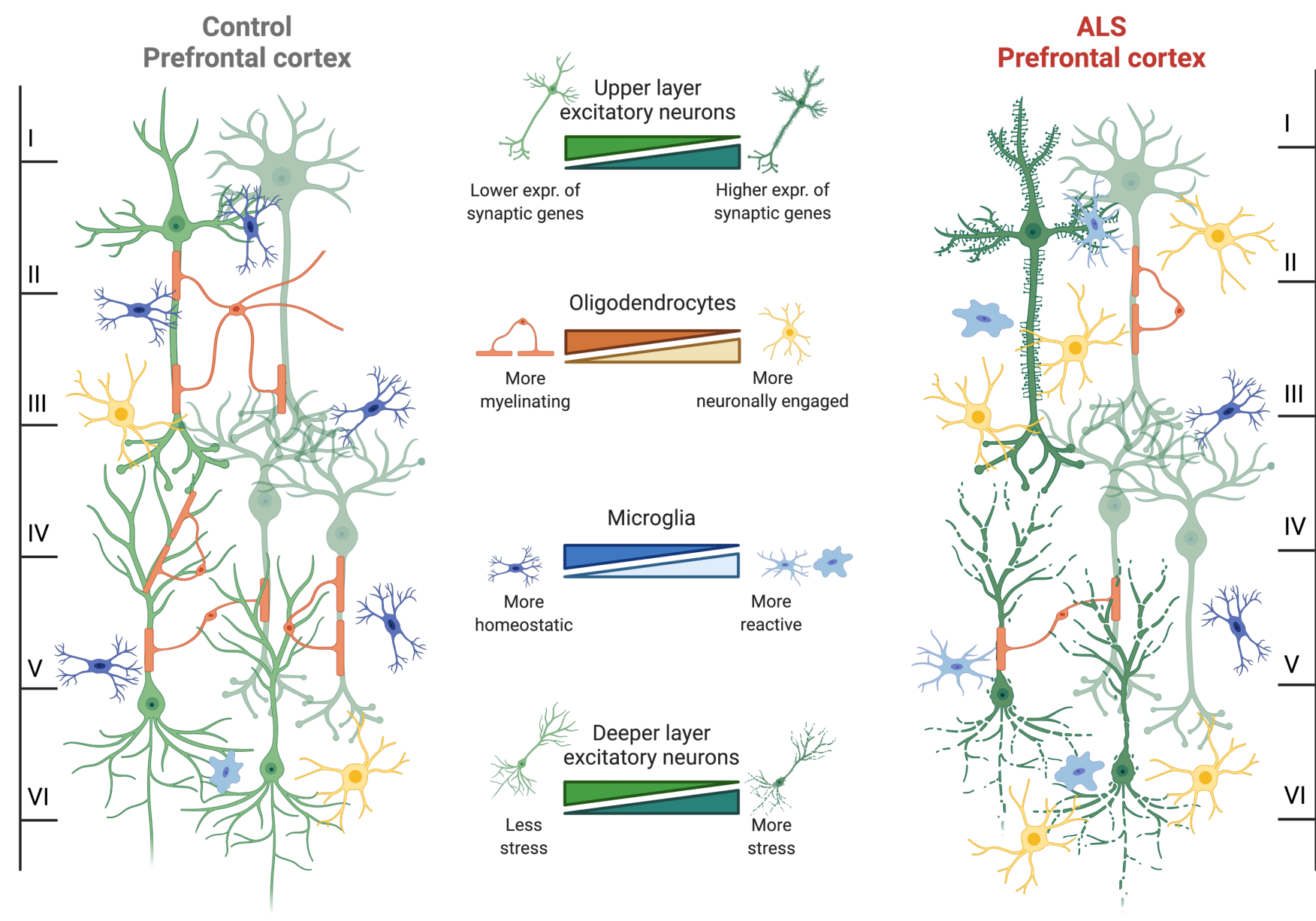

Graphical abstract and working model. Our study highlights cell type specific changes in prefrontal cortex of sporadic ALS patients. Specifically, we identify upregulation of synaptic molecules in excitatory neurons of upper cortical layers, interestingly correlating to hyperexcitability phenotypes seen in patients. Moreover, excitatory neurons of the deeper layers of the cortex, that project to the spinal cord and are most affected by the disease, show higher levels of cellular stresses than other neuronal types. Correspondently, oligodendrocytes transition from a highly myelinating state to a more neuronally engaged state, probably to counteract stressed phenotypes seen in excitatory neurons. At the same time, microglia show a reactive state with specific upregulation of endo-lysosomal pathways. 
a

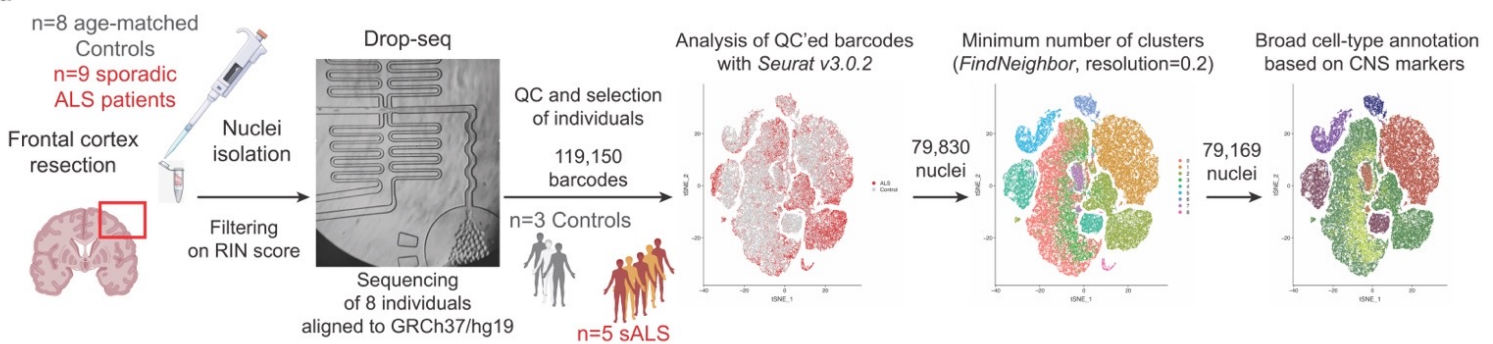

b
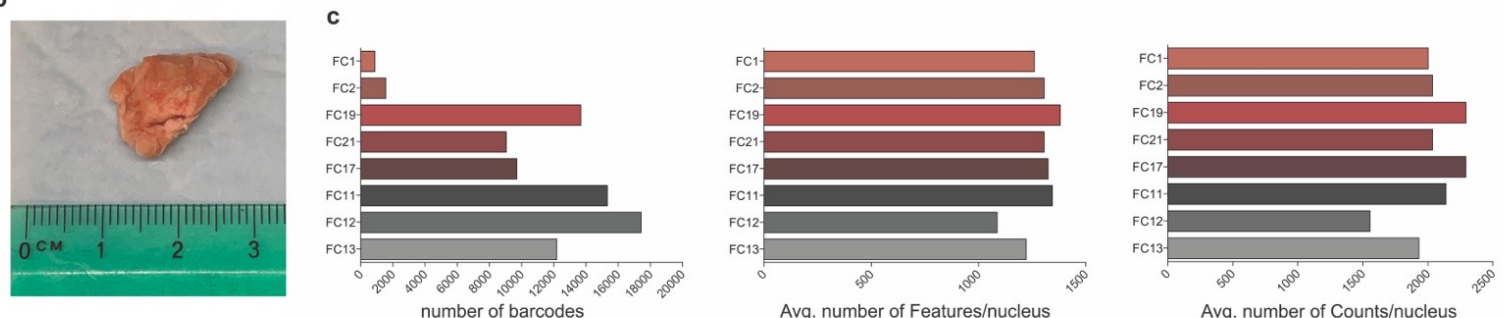

d
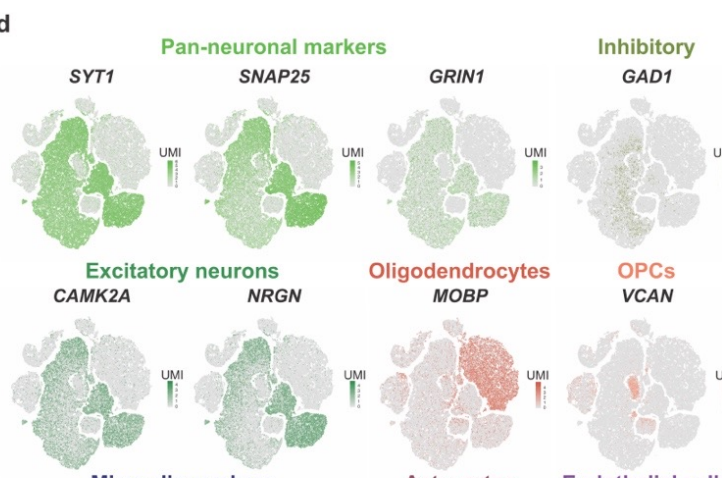

Microglia markers

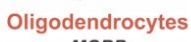

MOBP

VCAN
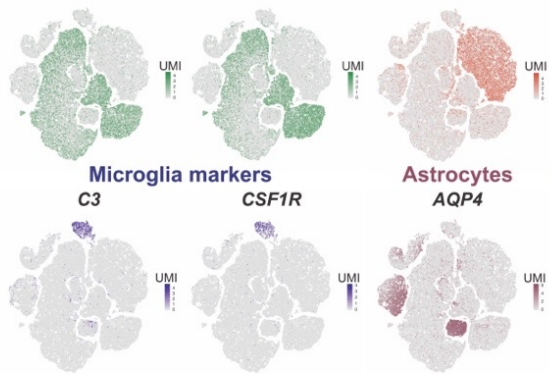

Astrocytes AQP4
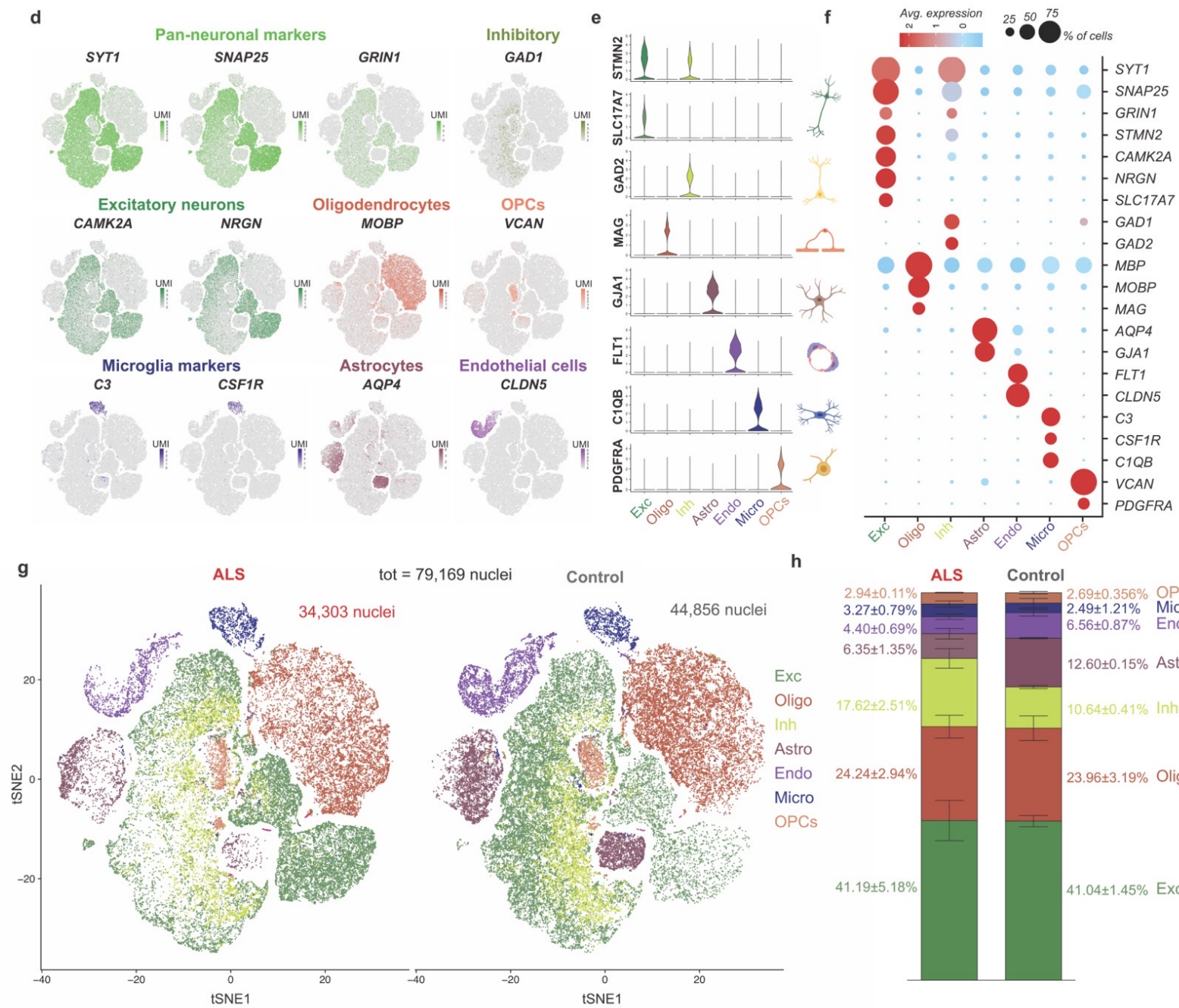

tot $=79,169$ nuclei

h

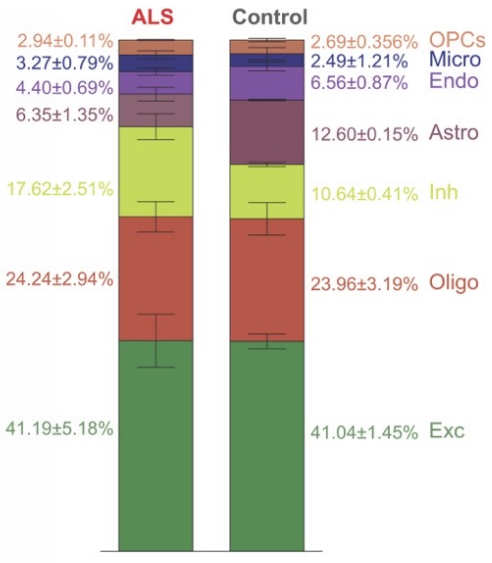

Extended Data Fig. 1 Technical parameters of snRNAseq and cell-type distribution across individuals a. Schematic diagram of cohort of sample and workflow for isolation of nuclei from cortices of ALS patients and agematched controls followed by single-cell RNA sequencing with DropSeq method, library generation and Quality Controls for analysis with Seurat 3.0.2 b. Frozen tissue from one of the individuals sequenced. c. Quality control parameter post-filtering per individual (FC - Frontal Cortex): number of total nuclei detected (barcodes), average number of genes detected per nucleus (nFeatures), and average number of UMIs (Unique Molecular Identifiers) per nucleus (nCounts). d. two-dimensional $t$-SNE projections of the whole cohort with expression of broad cell type markers e. Violin plots of selected cell type specific markers showing normalized gene expression (nUMIs). $\mathbf{f}$. Differential expression of additional cell type specific markers with percentage of cells expressing the given gene in each cluster. g. Two-dimensional $t$-SNE distribution of whole cohort with identified cell types annotations split by diagnosis (ALS patients $n=5$, age-matched Controls $n=3, n=79,169$ total nuclei). h. Fraction of each cell types identified in whole cohort split by diagnosis. 

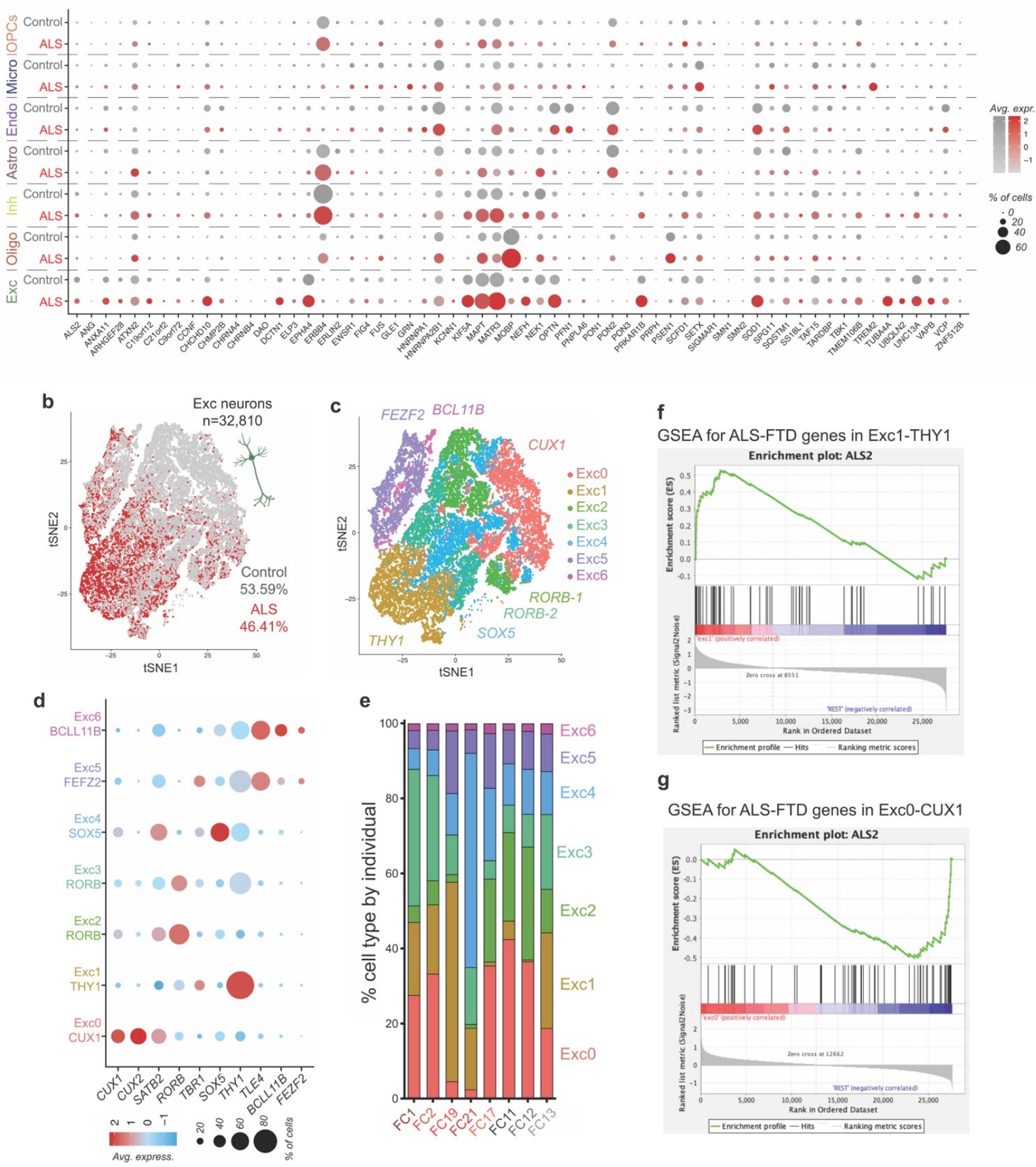

Extended Data Fig. 2 Expression of ALS-FTD associated genes in different cellular subtypes and excitatory neurons subtypes. a. Dotplot representing expression of gene associated with the ALS-FTD spectrum in each cell type identified in the whole cortex split by diagnosis. b. $t$-SNE projection of excitatory neurons cluster (ALS $n=15,227$ nuclei, Control $n=17,583$ nuclei). c. $t$-SNE projection of subclusters identified in excitatory neurons represents different, biologically relevant neuronal layers (FindNeighbor(res $=0.2)$ ). d. Dotplot representing percentage of cells expressing broad markers for different cortical layers. e. Distribution of excitatory neurons within subclusters by individual. f. Get Set Enrichment Analysis for the ALS-FTD associated genes in the lower THY1 excitatory neurons. g. Get Set Enrichment Analysis for the ALS-FTD associated genes in the upper CUX1 cortical neurons. 
822 genes up in all Exc neurons

123 genes up in THY1 exc1 ALS

234 genesup in CUX1 exc0 ALS

368 genes up in FEZF2 exc5 ALS
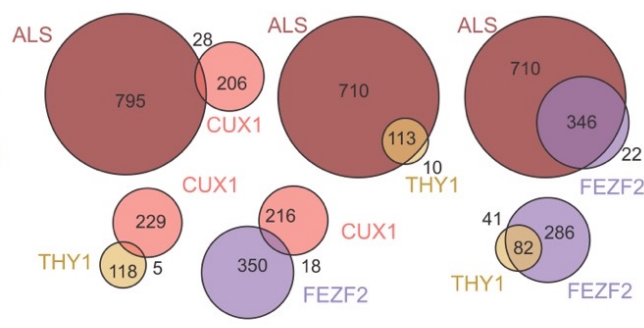

b GO terms for genes globally upregulated in ALS excitatory neurons (DGEall)

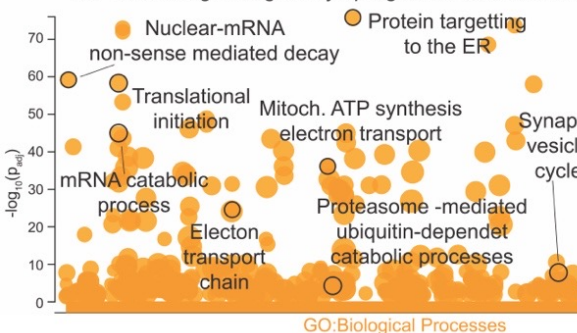

C protein targetting to ER GO::0045047

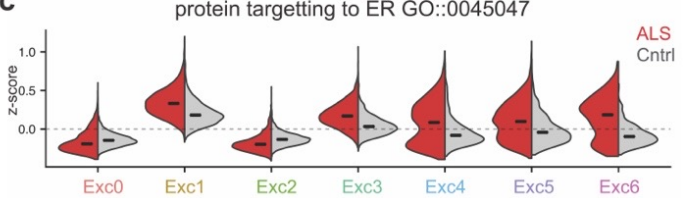
electron transport chain GO::0022900

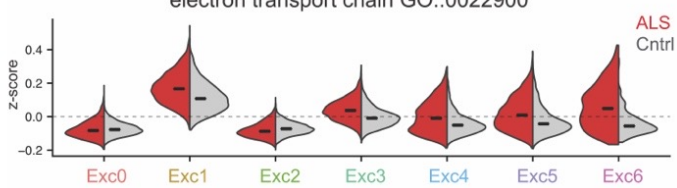

regulation of apoptotic signalling pathway GO::2001233
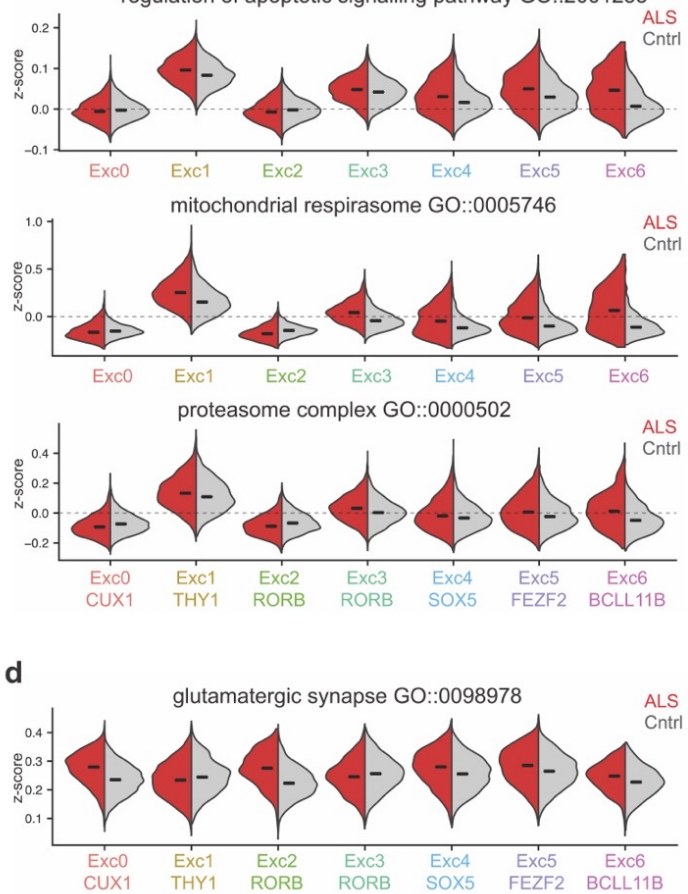

Cytosolic ribosome Respiratory

chain complexes

(1)
GO::0045047

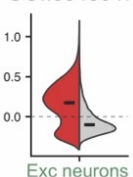

GO::0022900

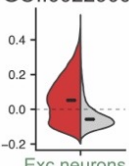

..2001233
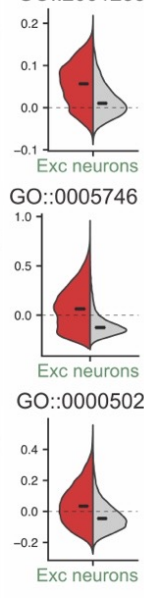

GO::0098978

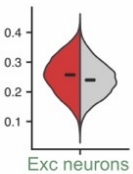

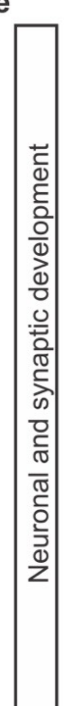
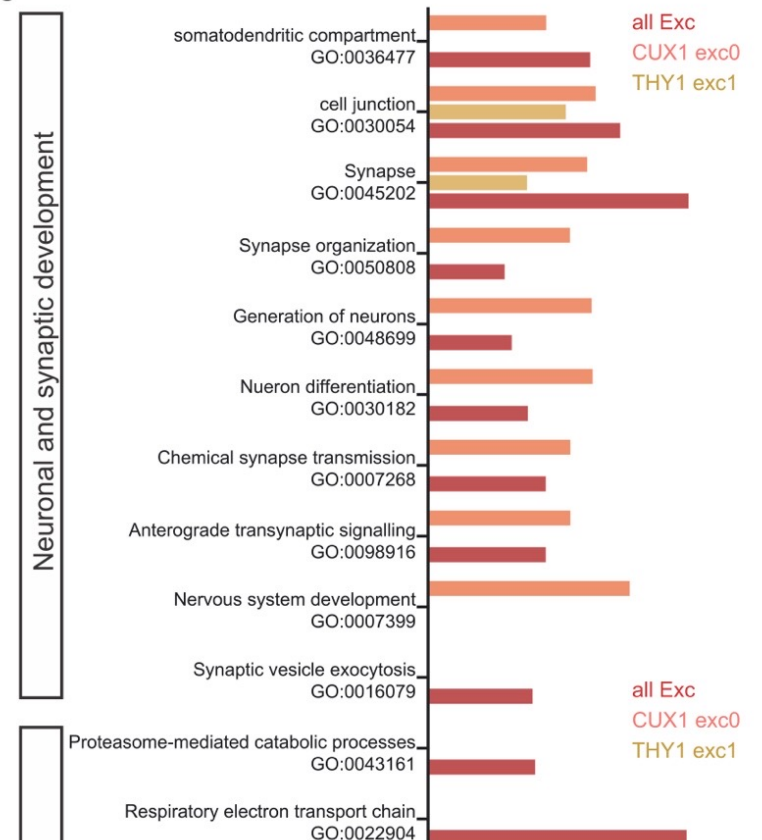

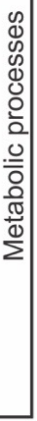

Anterograde transynaptic signalling GO:0098916

Nervous system development GO:0007399

exocytosis

GO:0016079

Apoptotic processes_-

GO:0008637

Respiratory chain complex I__ GO:0045271

Mitochondrial respiratory chain complex 1 GO:0005747

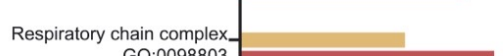

GO:0098803

Mitochondiral respirasome GO:0005746

Respirasome GO:0070469

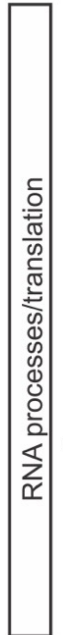

Ribosome GO:0005840

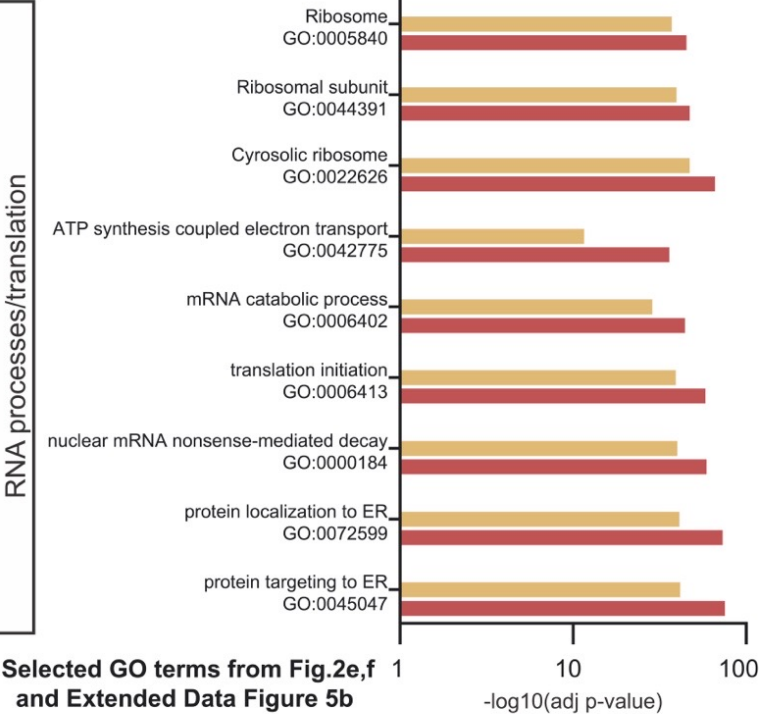
and Extended Data Figure 5b

$-\log 10(\operatorname{adj} p$-value)

Extended Data Fig. 3 Neurons of lower cortical layers express higher levels of stress pathways. a. Comparison of genes globally upregulated in ALS excitatory neurons (Exc all) with genes upregulated in specific layers: CUX2-exc0, THY1-exc1, FEZF2-exc5 (genes defined as expressed by $>10 \%$ of cells, 2-FC higher than Control, adjusted pvalue $<0.05)$. b. Gene Ontology analysis of terms associated with genes globally upregulated in excitatory neurons of ALS patients independently of groups (DGEall). c-d. Violin plots representing z-scores for selected, statistically significant GO terms upregulated in lower (c) layers and upper layers (d), in each subgroup (left) and globally (right). e. Representation of - $\log 10$ (adjusted p-vaules) of selected GO terms from previous figures for CUX2-Exc0 and THY1-Exc1 groups and globally. 


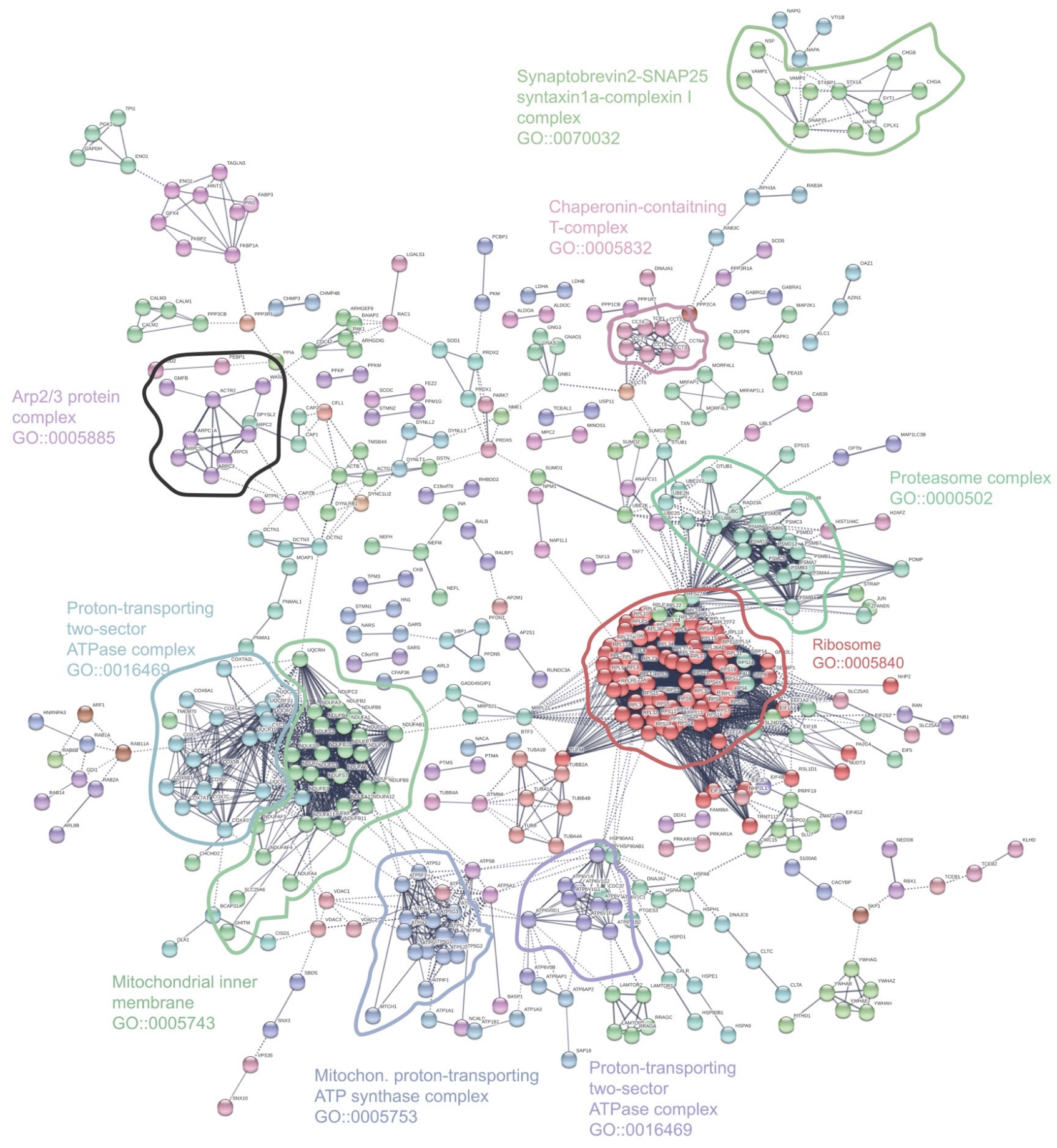

Extended Data Fig. 4 Global protein-protein interaction network for genes upregulated in ALS excitatory neurons. Color-coding derived from MLC clustering (4) to identified closely related groups of proteins. 
a

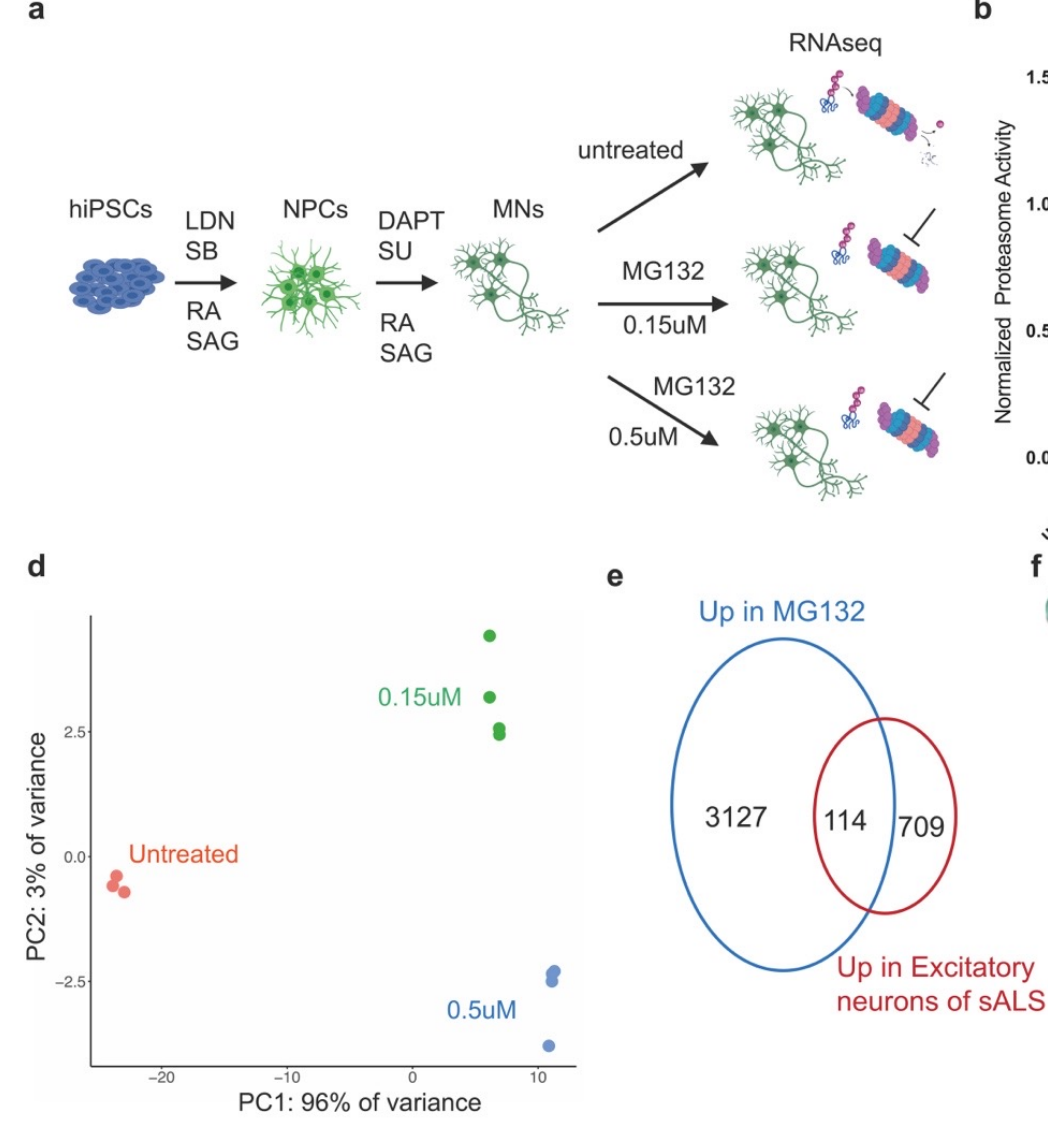

g

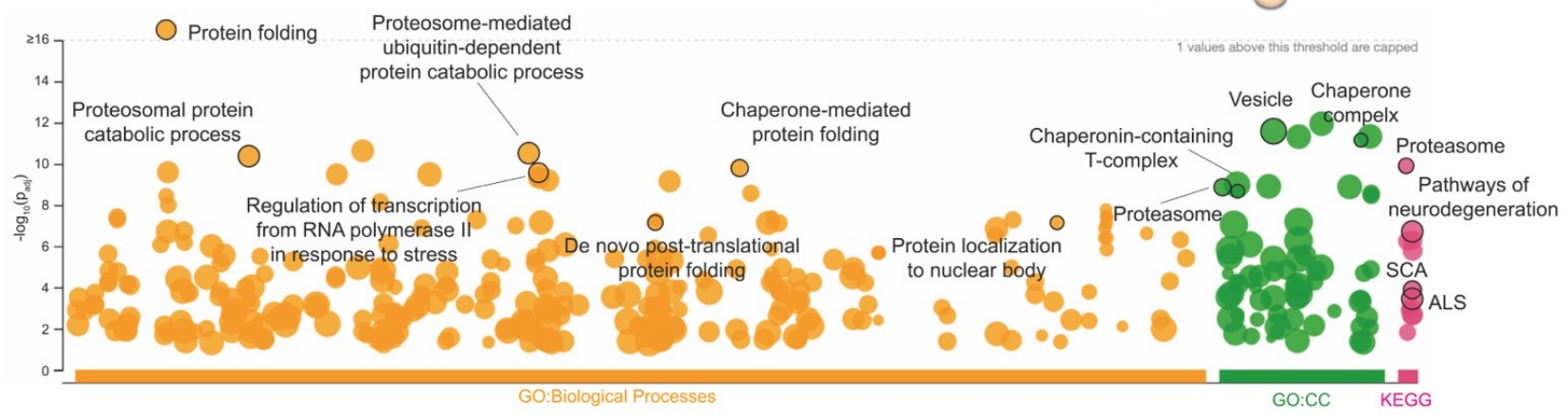

Extended Data Fig. 5 Proteostatic stress in hPSC-derived neurons resembles changes in excitatory neurons from brain of ALS patients. a. Diagram of neuronal differentiation from Pluripotent Stem Cells and treatment with proteasome inhibitors for bulk RNA-sequencing. b. Quantification of proteasome inhibition. c. Immunofluorescence of TDP-43 localisation after treatment. d. Principle Component Analysis plot showing strong effect of treatments compared to untreated controls. e. Venn Diagram depicting shared upregulated genes between treated hPSC-derived neurons and excitatory neurons from ALS patients. f. Protein-protein interaction network of shared genes from (d). g. Gene Ontology analysis for shared genes in (e), highlighted terms involved in protein folding and neurodegenerative diseases $(\mathrm{CC}=$ Cellular Components $)$.
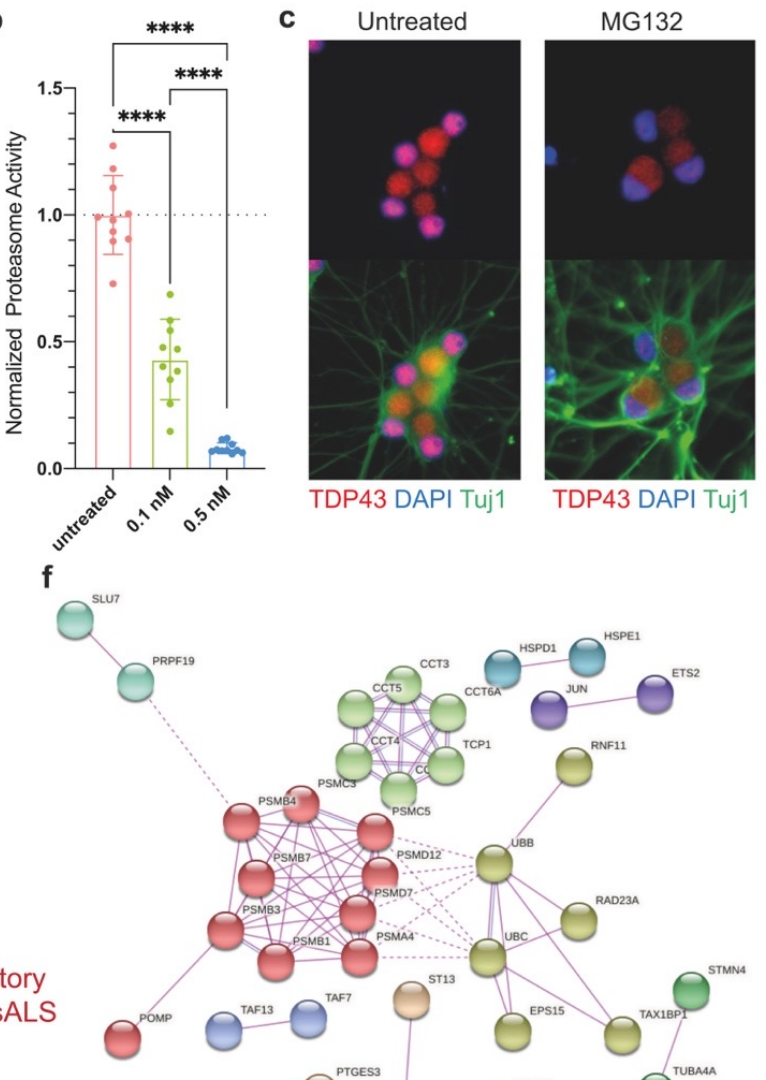

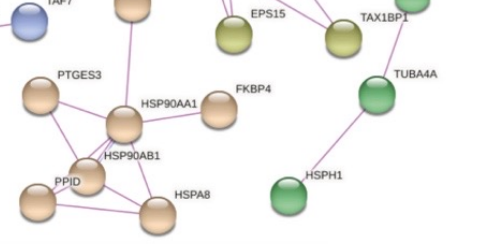



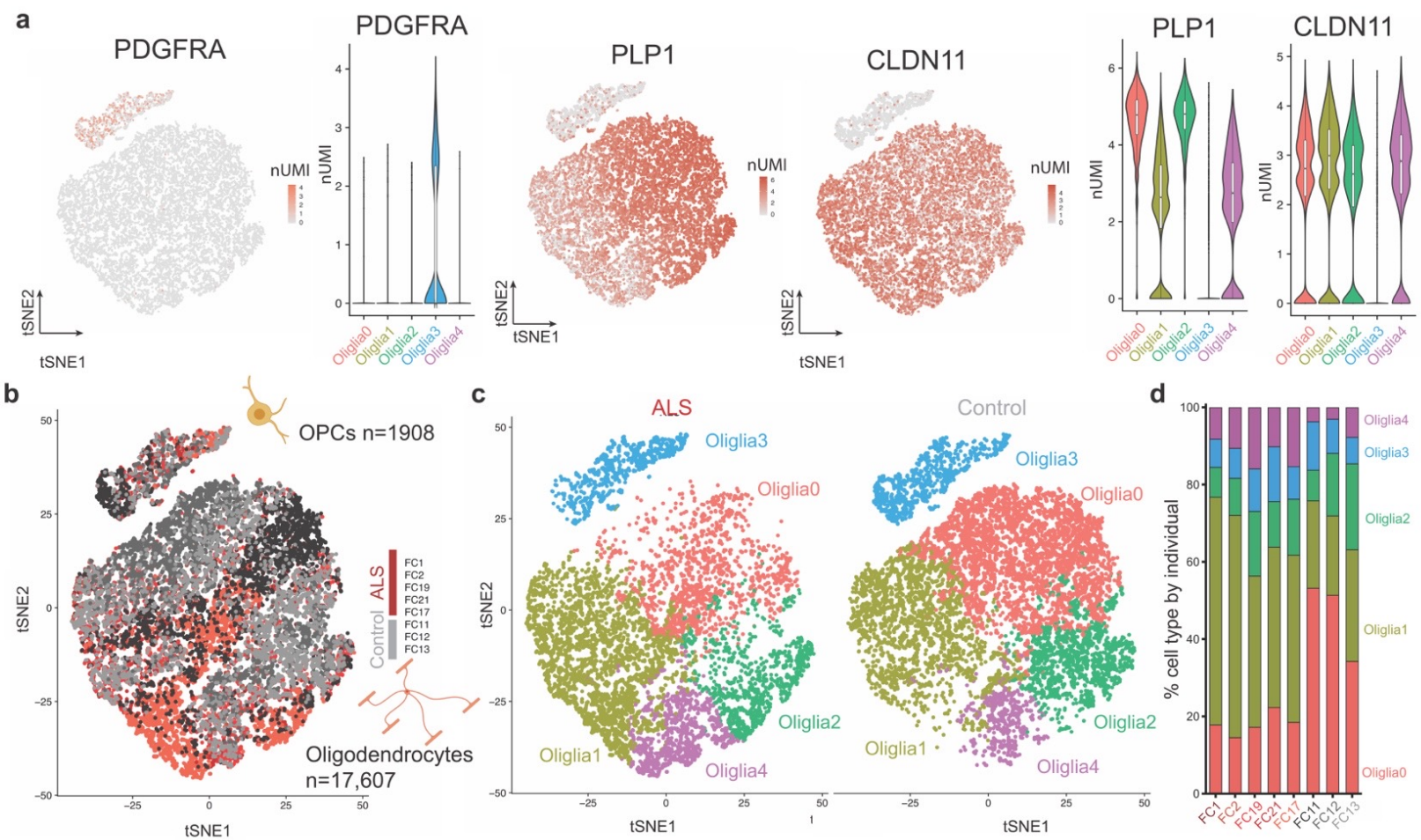

Extended Data Fig. 6 Distribution of oligodendroglial subtypes. a. $t$-SNE projection and corresponding Violin plots of additional broad markers of Oligodendrocyte Progenitor Cells (OPCs) and of mature oligodendrocytes. b. $t$-SNE projection of oligondedroglial cells by individual. c. $t$-SNE projection of subclusters identified within oligodendroglia split by diagnosis (FindNeighbor(res=0.2)). d. Distribution of oligodendroglia within subclusters by individual. 

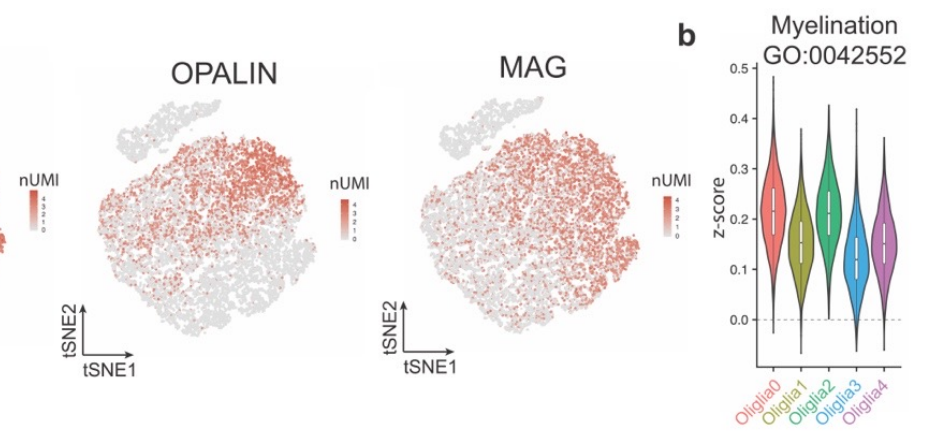

c

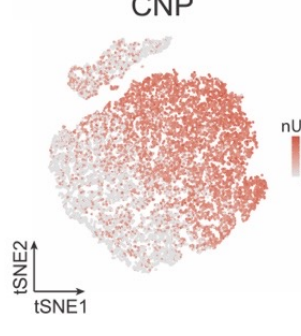

\section{DLG1}

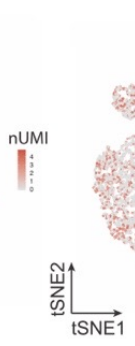

GRID2

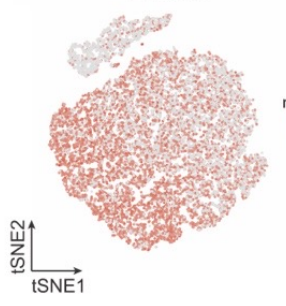

d
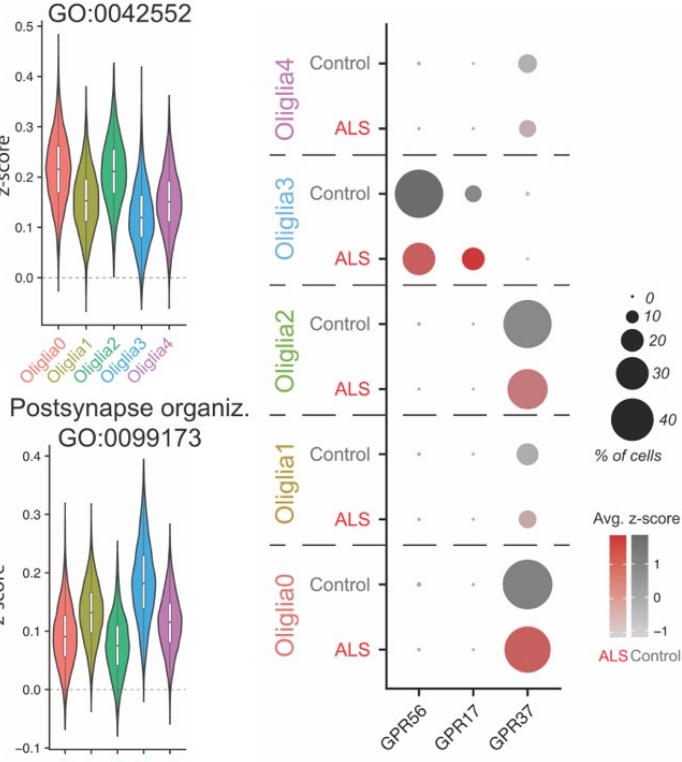

f
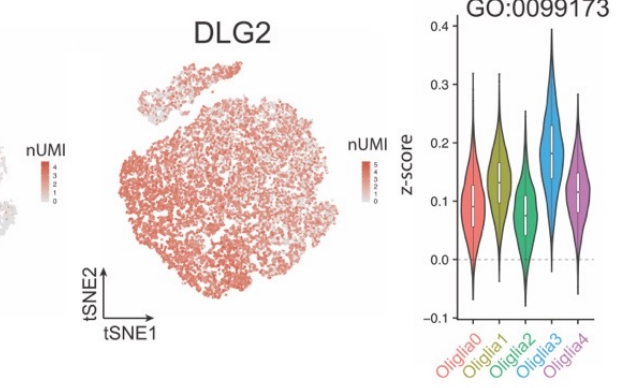

g

GO terms for genes upregulated in ALS
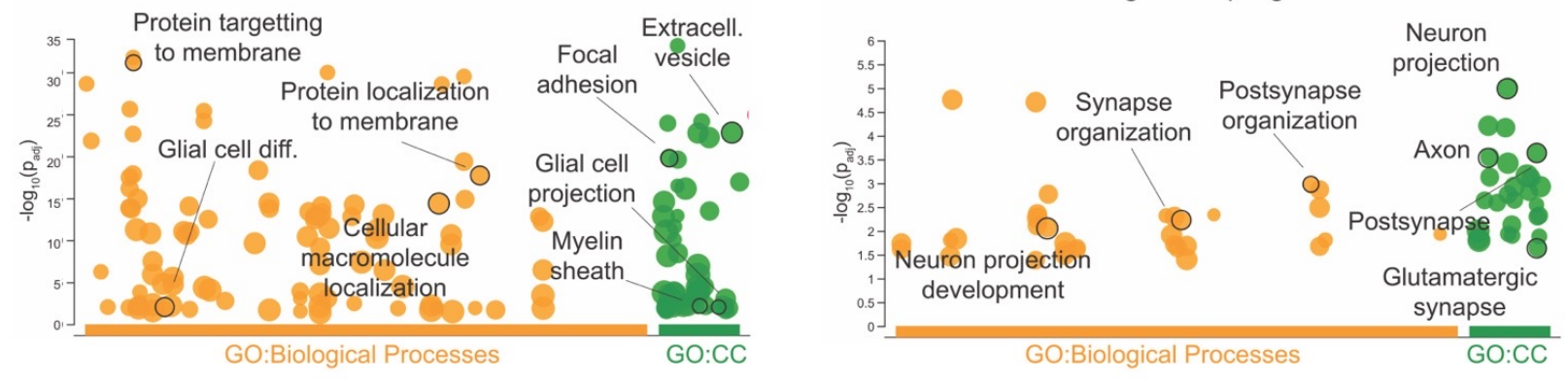

Extended Data Fig. 7 Oligodendrocytes polarization between myelinating and neurotrophic. a. $t$-SNE projection of broad markers of actively myelinating oligodendrocytes. b. $t$-SNE projection of broad markers of neuro-supportive oligodendrocytes. c,d. Violin plots representing relevant z-score for selected GO terms by cluster. e. Dotplot representing genes characteristic of maturation and development of OPCs into highly myelinating oligodendrocytes in each subcluster split by diagnosis. f. Gene Ontology analysis of terms associated with genes downregulated in ALS oligodendrocytes, highlighted terms involved in myelination (CC=Cellular Component). g. Gene Ontology analysis of terms associated with genes upregulated in ALS oligodendrocytes, highlighted terms involved in neuro-supportive functions $(\mathrm{CC}=$ Cellular Component. 

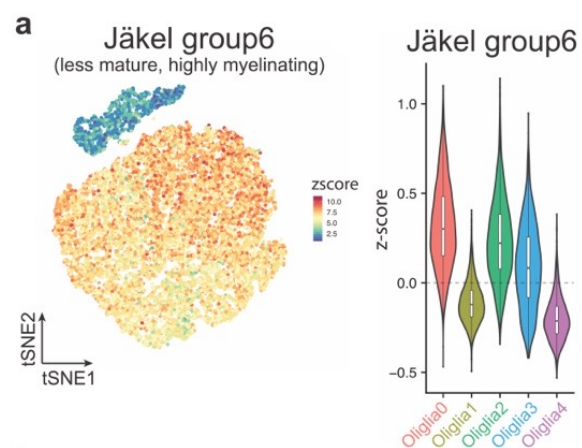

b
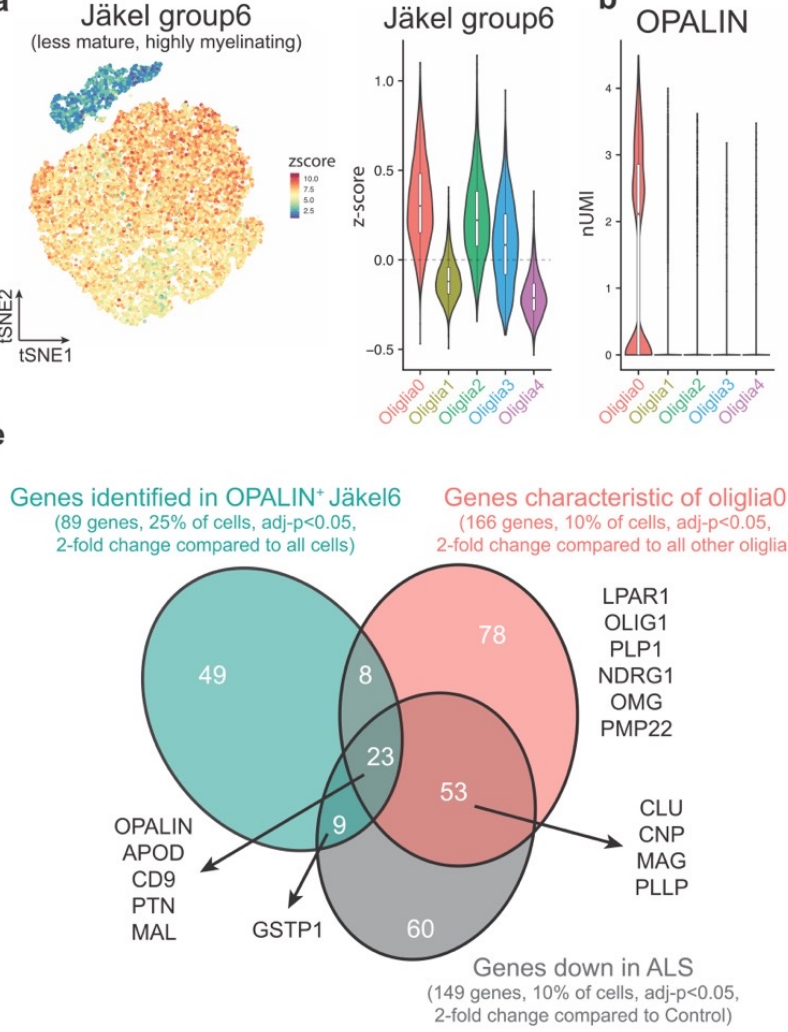

g

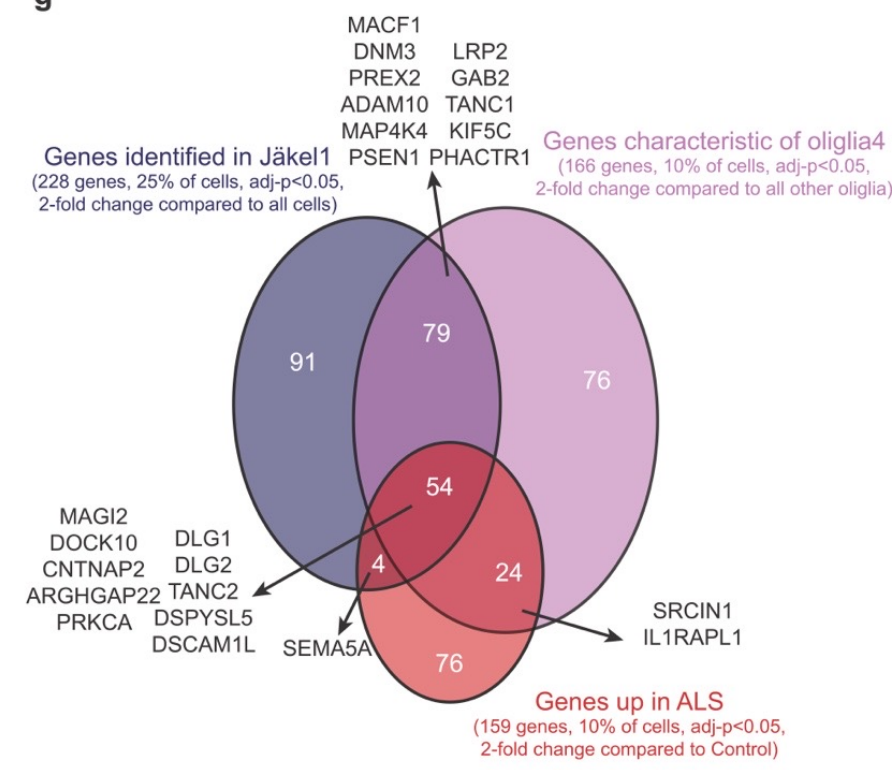

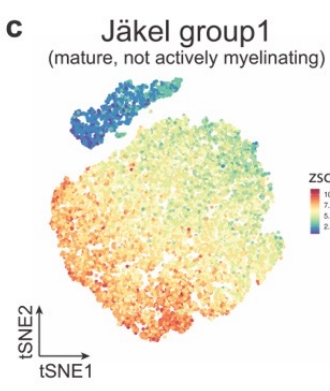
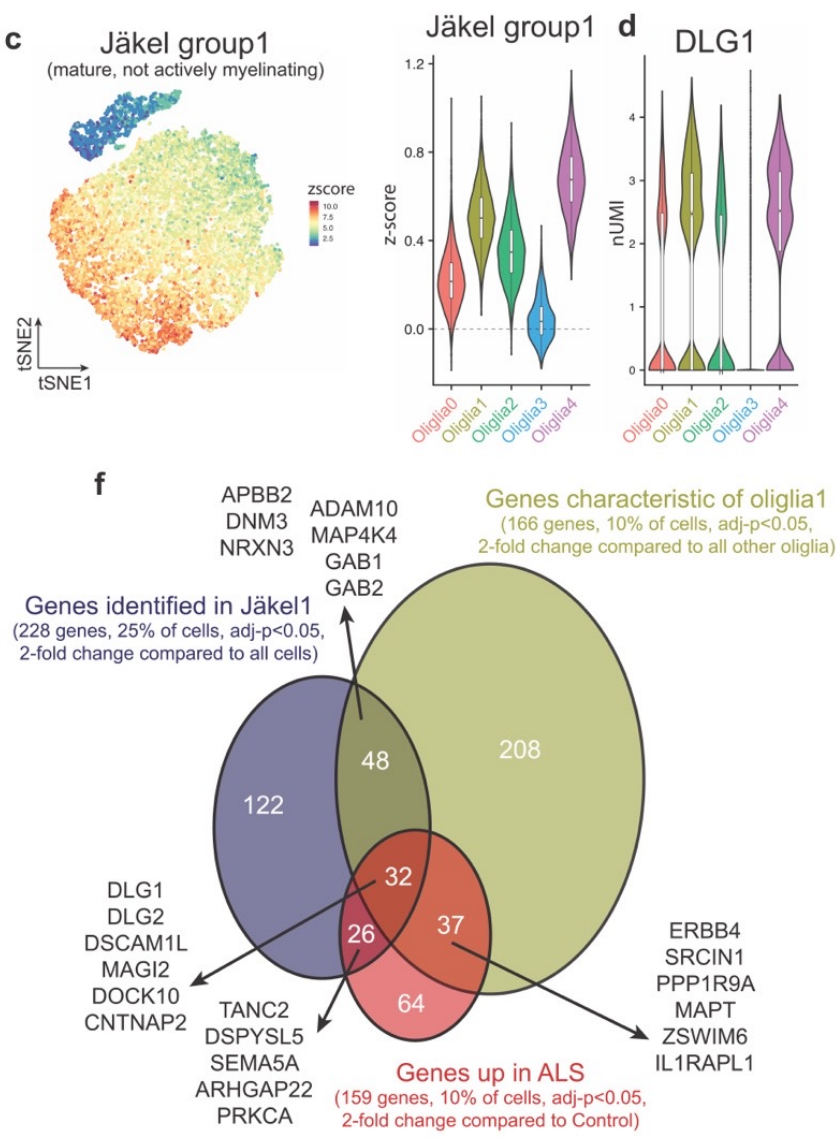

h

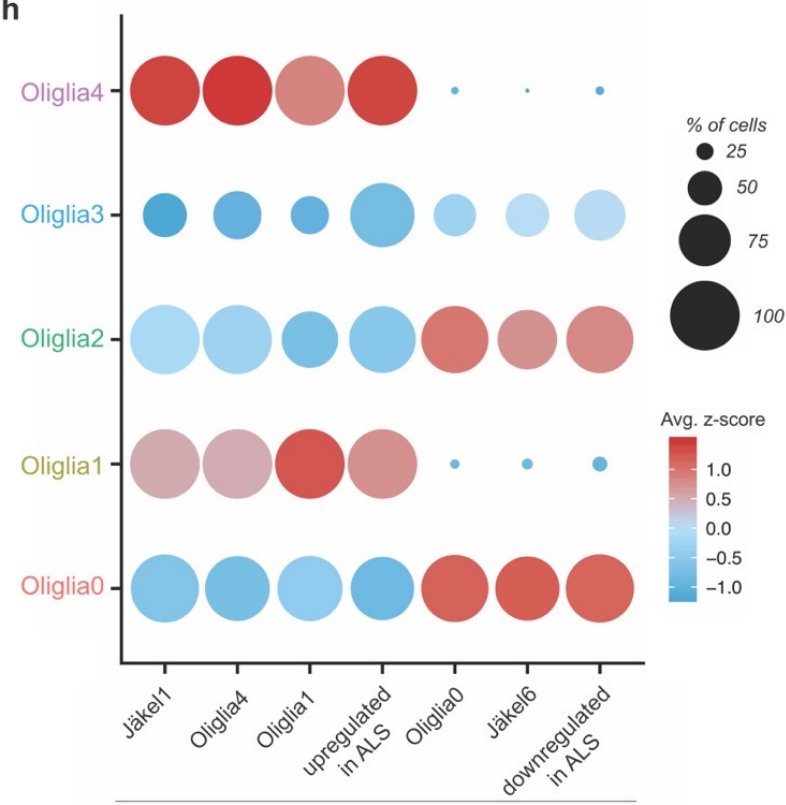

Z-scores for genetic signatures identified in:

Extended Data Fig. 8 Comparison of ALS-driven changes with other studies identified similar signatures disrupted in the disease. a. $t$-SNE projection and violin plot representing z-score for genes characteristic of highly myelinating, OPALIN ${ }^{+}$oligodendrocytes in Jäkel et al. b. Violin plot showing OPALIN expression in our dataset. c. $t$ SNE projection and violin plot representing z-score for genes of mature, not-actively myelinating oligodendrocytes in Jäkel et al. d. Violin plot showing DLG1 expression in our dataset. e. Comparison of genes downregulated in oligodendroglia from ALS patients with genes characteristic of highly myelinating, OPALIN ${ }^{+}$subtypes identified by this study (oliglia0) and by Jäkel et al (Jäkel6), highlighted genes are shared with GO terms shown in figures. f,g. Comparison of genes upregulated in oligodendroglia from ALS patients with genes characteristic of mature, lowly myelinating groups in this study (oliglia1 and 4) and by Jäkel et al (Jäkel1), highlighted genes are shared with GO terms shown in figures. h. Dotplot representing z-scores for the genetic signatures identified in the actively myelinating cells, the mature lowly myelinating cells and DEGs identified in this study. 
a

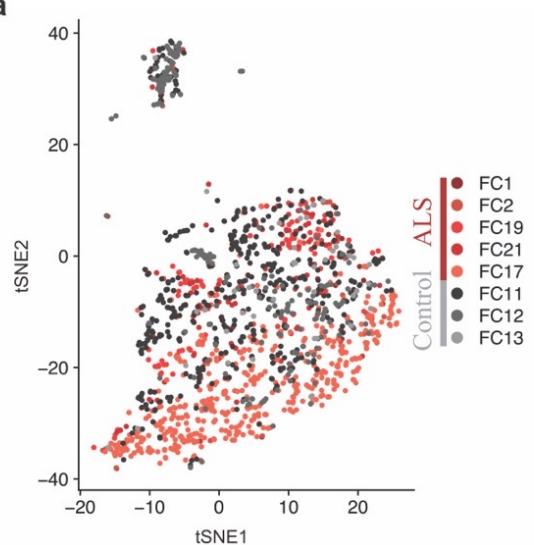

。

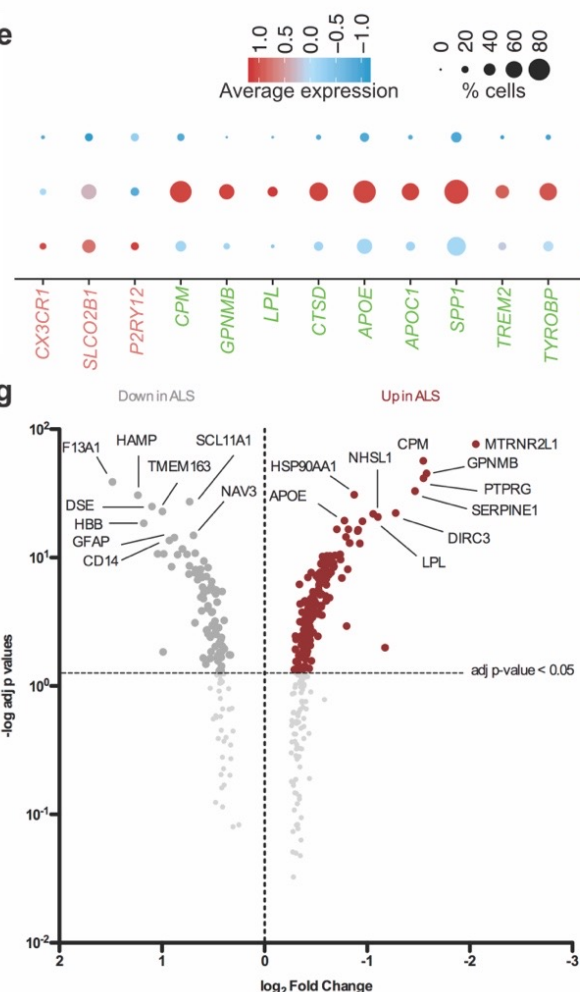

b

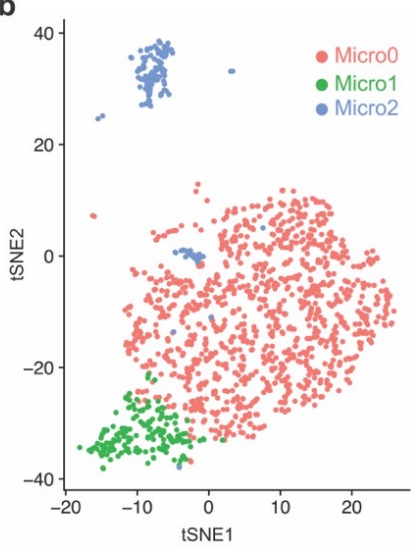

$\mathbf{f}$ c

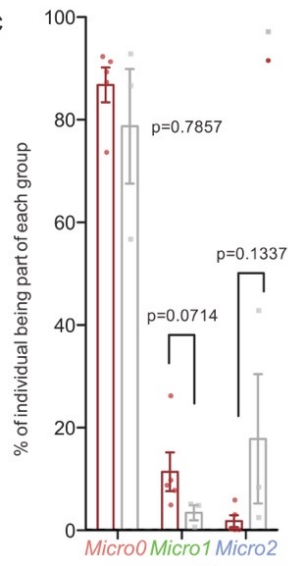

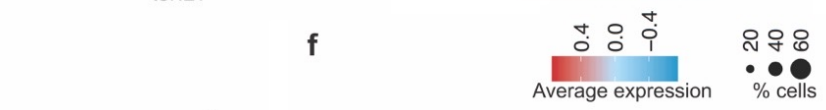
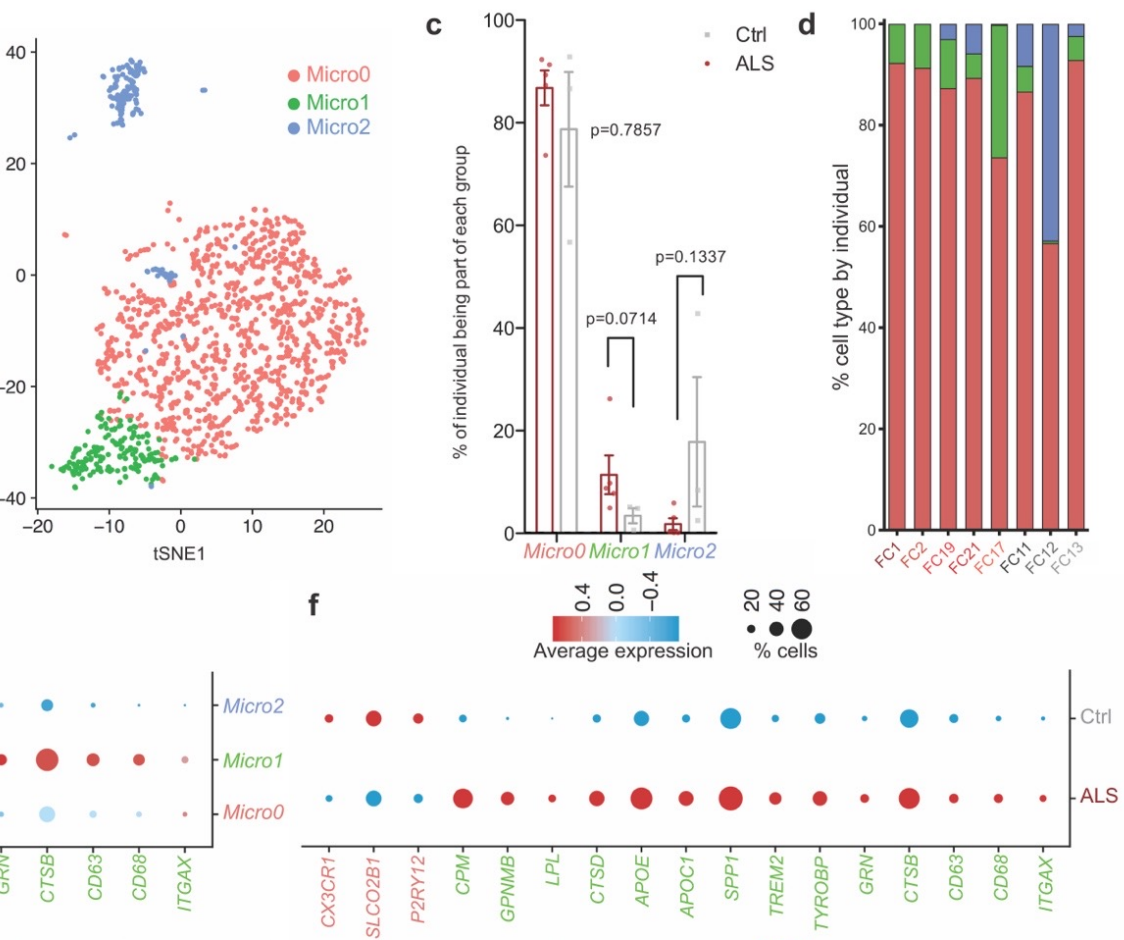

h

i 40

GO terms for DAMs-like Micro1

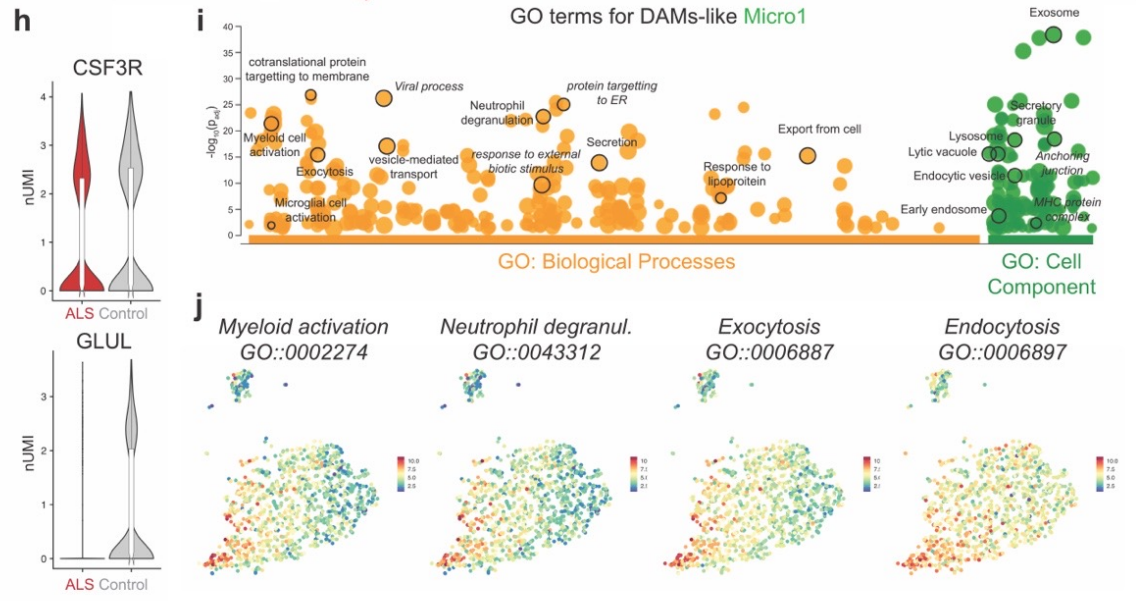

Extended Data Fig. 9 Shared features between ALS-driven changes and reactive subcluster of microglia. a. $t$-SNE projection of microglia by individual. b. $t$-SNE projection of subclusters identified within microglia (Micro0 $=$ Homeo $=$ homeostatic, Micro1=DAMs=Disease-associated microglia, Micro2=Cycling cells)). c. Distribution of microglia within clusters by diagnosis. d. Distribution of microglia within subclusters by individual. e. Dotplot representing genes identified as characteristic of Homeostatic microglia and DAMs by subcluster. f. Dotplot representing genes identified as characteristic of Homeostatic microglia and DAMs by diagnosis. g. Volcano plot of statistically significant differentially expressed genes between Control and ALS microglia (top ten upregulated and top ten downregulated genes highlighted). h. Violin plots of representative DEGs downregulated in ALS patients of genes associated with homeostatic microglia. i. Gene Ontology analysis of terms associated with genes characteristic of DAMs microglia, highlighted terms playing important role in microglial biology and/or pathogenesis of the disease. $\mathbf{j}$. $t$-SNE projections representing z-score for selected, statistically significant GO terms. 
a
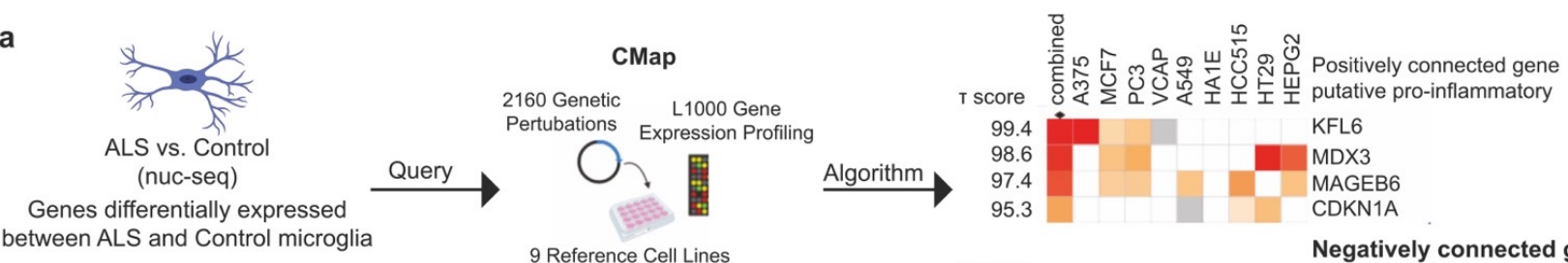

between ALS and Control microglia

9 Reference Cell Lines

T score

Negatively connected gene putative anti-inflammatory

IFNB1* *Potential off-the-shelf Deliverable Therapy

b
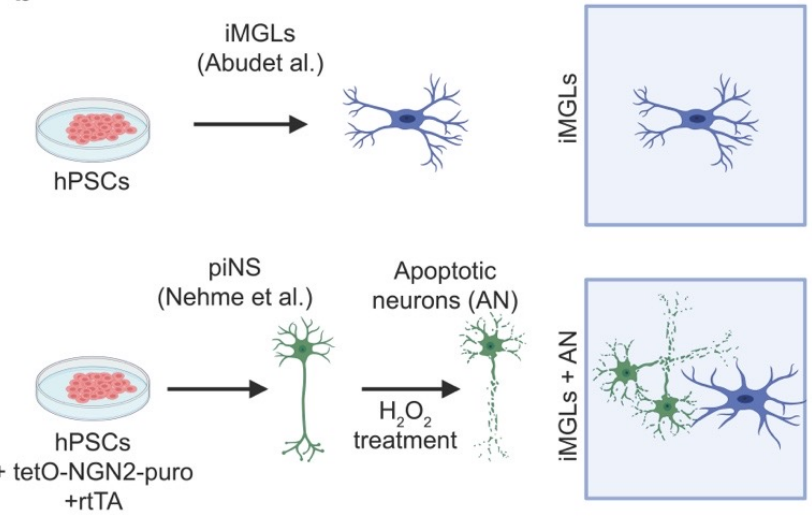

c

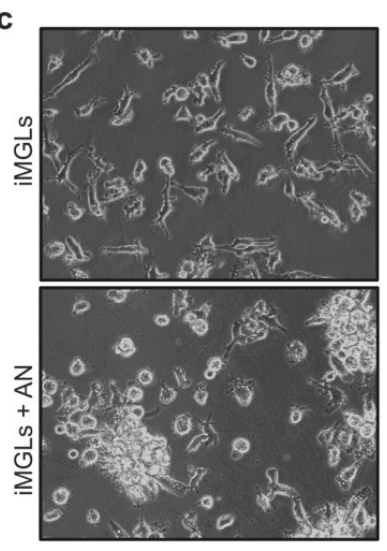

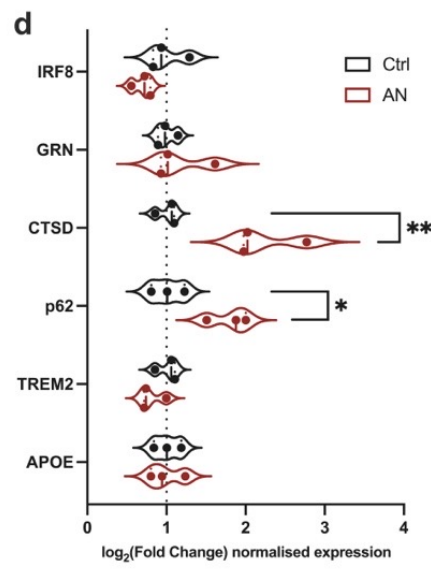

Extended Data Fig. 10 Apoptotic neurons upregulate lysosomal genes in microglia. a. Schematic of workflow and results from the Connectivity Map project for the genes upregulated in ALS microglia. Heatmap shows what cellular signature is most closely related to the query. b. Diagram of microglia and neuronal differentiation from Pluripotent Stem Cells, induction of apoptosis neurons and feeding to iMGLs. c. Brightfield images of untreated day 40 iMGLs and day 40 iMGLs fed apoptotic neurons for 24 hours. d. RT-qPCR quantification of ALS-driven genes after feeding apoptotic neurons to iMGLs. 